\title{
Multi-attribute group decision-making method based on weighted partitioned Maclaurin symmetric mean operator and a novel score function under neutrosophic cubic environment
}

\section{Jianping Fan}

Shanxi University

Shanshan Zhai

Shanxi University

Meiqin Wu ( $\nabla$ wmq80@sxu.edu.cn )

Shanxi University https://orcid.org/0000-0001-6126-1122

\section{Research Article}

Keywords: multi-attribute group decision-making (MAGDM), neutrosophic cubic set (NCS), weighted partitioned Maclaurin symmetric mean (WPMSM) operator, score function

Posted Date: May 3rd, 2021

DOl: https://doi.org/10.21203/rs.3.rs-332842/v1

License: (c) (1) This work is licensed under a Creative Commons Attribution 4.0 International License.

Read Full License 


\title{
Multi-attribute group decision-making method based on weighted partitioned Maclaurin symmetric mean operator and a novel score function under neutrosophic cubic environment
}

\author{
Jianping $\mathrm{Fan}^{1} \cdot$ Shanshan $\mathrm{Zhai}^{2} \cdot \mathrm{Meiqin} \mathrm{Wu}^{3}$ \\ School of Economics and Management, Shanxi University, Taiyuan 030006, China
}

\begin{abstract}
Neutrosophic cubic set (NCS) is the generalized version of neutrosophic sets and interval neutrosophic sets. It can deal with the complex information by combining the neutrosophic set (NS) and cubic set (CS). The partitioned Maclaurin symmetric mean (PMSM) operator can reflect the interrelationships among attributes where there are interrelationships among attributes in the same partition, but the attributes in different partitions are irrelevant. To effectively gather neutrosophic cubic information, we extend the PMSM operator to neutrosophic cubic environment and define the neutrosophic cubic partitioned Maclaurin symmetric mean (NCPMSM) operator and neutrosophic cubic weighted partitioned Maclaurin symmetric mean (NCWPMSM) operator. Later, we define a novel score function of NCS which overcome the drawbacks of the existing score functions. Next, based on NCWPMSM operator and the novel score function, we develop a multi-attribute group decision-making method. Finally, we give an example of supplier selection to illustrate the usefulness of the proposed multi-attribute group decision-making (MAGDM) method. At the same time, a comparative analysis is to show the effectiveness and advantages of the proposed method compared with the existing methods.
\end{abstract}

Keywords multi-attribute group decision-making (MAGDM) $\cdot$ neutrosophic cubic set (NCS) $\cdot$ weighted partitioned Maclaurin symmetric mean (WPMSM) operator $\cdot$ score function

\section{Introduction}

The entire world is designated with complex circumstances. In order to process the complexity

\section{$\triangle$ Jianping Fan \\ wmq80@sxu.edu.cn}

${ }^{1}$ School of Economics and Management, Shanxi University, Taiyuan 030000, China and uncertainty, Zadeh (1965) firstly introduced the concept of fuzzy set (FS) in 1965. FS represents the uncertainty of decision information by the membership degree which is in the closed interval $[0,1]$. However, in the process of cognition, people tend to hesitate in different degrees or show a certain degree of lack of knowledge, so the cognitive results are shown as positive, negative or intermediate between positive and negative. In order to 
overcome this shortcoming, Atanassov (1986, 1989) proposed the concept of intuitionistic fuzzy set (IFS) in 1986. IFS considers both membership information and non-membership information at the same time, so it has a stronger performance in dealing with uncertain information. However, in some real situations, the membership degree, non-membership degree and hesitation of elements may not be specific values, so IFS was extended to interval-value intuitionistic fuzzy set (IVIFS) by Atanassov and Gargov (1989). In IFS and IVIFS, the membership degree and non-membership degree are defined independently, while hesitant degree is dependent on membership degree and non-membership degree. To overcome this limitation, Smarandache (1999) proposed the concept of neutrosophic set (NS). NS includes membership degree $\mathrm{T}(\mathrm{x})$, indeterminacy degree $\mathrm{I}(\mathrm{x})$ and non-membership degree $\mathrm{F}(\mathrm{x})$ of elements. Wang and Zhang et al. (2005) further proposed the concept of interval neutrosophic set (INS), where the representation of the $\mathrm{T}(\mathrm{x}), \mathrm{I}(\mathrm{x})$ and $\mathrm{F}(\mathrm{x})$ extended from single values to interval values. Wang et al. (2010) proposed the single-valued neutrosophic set (SVNS) theory.

In 2012, Jun et al. (2011) proposed the concept of cubic set (CS), which is characterized by intervalvalue fuzzy set (IVFS) (Turksen 1986) and FS. CS can contain more information and it is more effective to deal with uncertain and vague information. Mehmood et al. (2016) proposed the cubic hesitant fuzzy sets (CHFSs). Muhiuddin and Al-roqi (2014) proposed the cubic soft sets (CSSs). Aslam et al. (2020) proposed develop trapezoidal cubic linguistic uncertain fuzzy numbers (TrCLUFNs), and propose the trapezoidal cubic linguistic uncertain fuzzy Einstein weighted average (TrCLUFEWA) operator and the trapezoidal cubic linguistic uncertainty fuzzy Einstein mixed weighted average (TrCLUFEHWA) operator to aggregate trapezoidal cubic linguistic uncertain fuzzy information. Amin et al. (2020) introduced six uncertain fuzzy operators based on triangular cubic linguistic information, and proposed a multi-attribute group decision-making method. Jun et al. (2017) introduced the cubic set to neutrosophic set and proposed the concept of neutrosophic cubic set (NCS). NCS has attracted the attention of many researchers, because it is more effective and informative by the available information in the form of INS and SVNS. Gulistan et al. (2018) combined NCS and Molodtsov's soft sets (1999) with matrices to develop a neutrosophic cubic soft matrix theory. Gulistan et al. (2019) combined NCS and complex fuzzy set and proposed the complex neutrosophic cubic set (internal and external).Cui et al. (2019) proposed the concept of dynamic neutrosophic cubic set (DNCS) to describe patients' disease symptoms in different time intervals and proposed the logarithmic similarity measure (LSM) of DNCSs to apply it to medical diagnosis.

Information integration is a common activity in multi-attribute decision-making. The Maclaurin symmetric mean (MSM) operator is one of the aggregation methods that proposed by Maclaurin (1729). The MSM operator fully considers the correlation among attributes. Qin et al. (2014) proposed the weighted intuitionistic fuzzy Maclaurin symmetric mean (WIFMSM) operator firstly by introducing the MSM to the fuzzy information aggregation field. $\mathrm{Li}$ et al. (2016) applied the Maclaurin symmetric mean operator to gather hesitant fuzzy information, and defined the weighted hesitant fuzzy Maclaurin symmetric mean (WHFMSM) operator for human resources management. Wang et al. (2018) made a study on the weighted single-valued neutrosophic linguistic Maclaurin symmetric mean (WSVNLMSM) operator and verified its properties. Based on the MSM operator and geometric mean (GM) operator, Qin et al. (2015) proposed the dual Maclaurin symmetric mean (DMSM) operator. The MSM and its extension can capture the correlation between multiple input values, but not all attributes are related. Based on this, Bai et al. (2018) proposed the concept of partitioned Maclaurin symmetric mean (PMSM) operator. The PMSM operator has several advantages in processing the situation where there are interrelationships among attributes in the same partition, but the attributes in different partitions are irrelevant. So we introduce the partitioned Maclaurin symmetric mean operator to neutrosophic cubic environment and proposed 
neutrosophic cubic partitioned Maclaurin symmetric mean (NCPMSM) operator and neutrosophic cubic weighted partitioned Maclaurin symmetric mean (NCWPMSM) operator.

The rest of this paper is shown below. The section II briefly introduces some theoretical basis for this paper. Section III introduces the NCPMSM operator, the NCWPMSM operator, and a novel score function of neutrosophic cubic numbers. In section IV, a multi-attribute group decision-making method based on the NCWPMSM operator and a novel score function is introduced. In section V, an example of supplier selection is introduced to illustrate the feasibility and superiority of the proposed method. Finally, section VI gives the conclusion.

\section{Preliminaries}

Definition 1. (Zadeh 1965) Let $X$ be an universal set and $\mathrm{x}$ be the element in $\mathrm{X}$. Then a FS F in $\mathrm{X}$ is defined as: $\mathrm{F}=\left\{<\mathrm{x}, \mu_{\mathrm{A}}(\mathrm{x})>\mid \mathrm{x} \in \mathrm{X}\right\}$, where $\mu_{\mathrm{A}}(\mathrm{x}): \mathrm{x} \rightarrow$ $[0,1]$ is the membership degree of the element $\mathrm{x} \in$ $\mathrm{X}$.

Definition 2. (Atanassov 1986; Atanassov 1989) Let $\mathrm{X}$ be an universal set and $\mathrm{x}$ be the element in $\mathrm{X}$. Then an IFS in $X$ is defined as: $A^{*}=\left\{<x, \mu_{A}(x), v\right.$ $\mathrm{A}(\mathrm{x})>\mid \mathrm{x} \in \mathrm{X}\}$, where $\mu_{\mathrm{A}}(\mathrm{x}): \mathrm{X} \rightarrow[0,1]$ and $\nu_{\mathrm{A}}(\mathrm{x}):$ $\mathrm{X} \rightarrow[0,1]$ with the condition $0 \leq \mu_{\mathrm{A}}(\mathrm{x})+\mathrm{V}_{\mathrm{A}}(\mathrm{x}) \leq 1$, $\forall \mathrm{x} \in \mathrm{X}$. The $\mu_{\mathrm{A}}(\mathrm{x})$ and $\nu_{\mathrm{A}}(\mathrm{x})$ represent the membership degree and non-membership degree of the element $x$ to the set $A^{*}$ respectively.

Definition 3. (Zeng et al. 2019) Let $d=[a, b]$ be an IFV, where $\mathrm{a} \in[0,1], \mathrm{b} \in[0,1]$ and $0 \leq \mathrm{a}+\mathrm{b} \leq 1$. Let $\pi_{\mathrm{d}}$ be the hesitant degree of the IFV d, where $\pi_{\mathrm{d}}$ $\in[0,1]$. Then the score function $\mathrm{S}_{\mathrm{CK}}(\mathrm{d})$ of the IFV $\mathrm{d}$ is defined as :

$$
\begin{aligned}
S_{C K}(d) & =a-b-(1-a-b) \times \frac{\log _{2}(2-a-b)}{100} \\
& =a-b-\pi_{d} \times \frac{\log _{2}(1+(1-a-b))}{100} \\
& =a-b-\pi_{d} \times \frac{\log _{2}\left(1+\pi_{d}\right)}{100},
\end{aligned}
$$

where $\mathrm{S}_{\mathrm{CK}}(\mathrm{d}) \in[-1,1]$. In the score function, the larger of the first part " $a-b$ ", the larger the score value $\mathrm{S}_{\mathrm{CK}}(\mathrm{d})$. The second part " $\pi_{\mathrm{d}} \times$ $\log _{2}\left(1+\pi_{\mathrm{d}}\right) / 100$ " is used to distinguish two IFVs which have the same value of " $a-b$ ", and the smaller the value of " $\pi_{\mathrm{d}} \times \log _{2}\left(1+\pi_{\mathrm{d}}\right) /$ 100 ", the larger the score value $\mathrm{S}_{\mathrm{CK}}(\mathrm{d})$ of the IFV d.

Definition 4. (Atanassov et al. 1999) Let X be a set of objects and $\mathrm{x}$ be the element in X. The NS $A$ in $X$ consists of $T_{A}(x)$ - membership degree, $I_{A}(x)$ - uncertainty degree and $F_{A}(x)$ - nonmembership degree, and it is defined as $A=\{<x$, $\left.\mathrm{T}_{\mathrm{A}}(\mathrm{x}), \mathrm{I}_{\mathrm{A}}(\mathrm{x}), \mathrm{F}_{\mathrm{A}}(\mathrm{x})>\mid \mathrm{x} \in \mathrm{X}\right\} . \mathrm{T}_{\mathrm{A}}(\mathrm{x}), \mathrm{I}_{\mathrm{A}}(\mathrm{x}), \mathrm{F}_{\mathrm{A}}(\mathrm{x})$ are non-standard subsets in $] 0^{-}, 1^{+}\left[\right.$, i.e. $\mathrm{T}_{\mathrm{A}}(\mathrm{x}): \mathrm{X}$ $\rightarrow] 0^{-}, 1^{+}\left[, \mathrm{I}_{\mathrm{A}}(\mathrm{x}): \mathrm{X} \rightarrow\right] 0^{-}, 1^{+}\left[\right.$, and $\mathrm{F}_{\mathrm{A}}(\mathrm{x}): \mathrm{X}$ $\rightarrow$ ]0-,1+[. Due to the sum of $\mathrm{T}_{\mathrm{A}}(\mathrm{x}), \mathrm{I}_{\mathrm{A}}(\mathrm{x})$ and $\mathrm{F}_{\mathrm{A}}(\mathrm{x})$ is unlimited, so $0^{-} \leq \mathrm{T}_{\mathrm{A}}(\mathrm{x})+\mathrm{I}_{\mathrm{A}}(\mathrm{x})+\mathrm{F}_{\mathrm{A}}(\mathrm{x})$ $\leq 3^{+}$.

Definition 5. (Wang et al. 2010) Let $X$ be a set of objects and $\mathrm{x}$ be the element in $\mathrm{X}$. When $\mathrm{T}_{\mathrm{A}}(\mathrm{x}), \mathrm{I}_{\mathrm{A}}(\mathrm{x})$ and $\mathrm{F}_{\mathrm{A}}(\mathrm{x})$ respectively degenerate to an exact number, then $\mathrm{A}$ is a SVNS.

Definition 6. (Wang et al. 2005) Let X be a set of objects and $\mathrm{x}$ be the element in X. The NS A on $\mathrm{X}$ consists of $\mathrm{T}_{\mathrm{A}}(\mathrm{x})$ - membership degree, $\mathrm{I}_{\mathrm{A}}(\mathrm{x})$ - uncertainty degree and $\mathrm{F}_{\mathrm{A}}(\mathrm{x})$ - nonmembership degree. When $\mathrm{T}_{\mathrm{A}}(\mathrm{x}), \mathrm{I}_{\mathrm{A}}(\mathrm{x})$ and $\mathrm{F}_{\mathrm{A}}(\mathrm{x})$ belong to closed interval $[0,1]$, i.e. $\mathrm{T}_{\mathrm{A}}(\mathrm{x}): \mathrm{X} \rightarrow$ $[0,1], \mathrm{I}_{\mathrm{A}}(\mathrm{x}): \mathrm{X} \rightarrow[0,1]$, and $\mathrm{F}_{\mathrm{A}}(\mathrm{x}): \mathrm{X} \rightarrow[0,1]$, then $\mathrm{A}$ is an INS which can be expressed as $\mathrm{A}=$ $\left\{\mathrm{x},\left[\mathrm{T}_{\mathrm{A}}^{\mathrm{L}}(\mathrm{x}), \mathrm{T}_{\mathrm{A}}^{\mathrm{U}}(\mathrm{x})\right],\left[\mathrm{I}_{\mathrm{A}}^{\mathrm{L}}(\mathrm{x}), \mathrm{I}_{\mathrm{A}}^{\mathrm{U}}(\mathrm{x})\right],\left[\mathrm{F}_{\mathrm{A}}^{\mathrm{L}}(\mathrm{x}), \mathrm{F}_{\mathrm{A}}^{\mathrm{U}}(\mathrm{x})\right]>\right.$ $\mid x \in X\}$. Similarly, the sum of $T_{A}(x), I_{A}(x)$ and $F_{A}(x)$ satisfies: $0 \leq T_{A}^{U}+I_{A}^{U}(x)+F_{A}^{U}(x) \leq 3$.

Definition 7. (JUN et al. 2011) let $X$ be an universe, then a CS can be expressed as the following form :

$$
\Omega=\{\langle x, \Psi(x), \mu(x)\rangle \mid x \in X\},
$$

in which $\Psi$ is an interval value fuzzy set (IVFS) and $\mu$ is a fuzzy set (FS).

The notion of NCS is an extension of CS.

Definition 8. (Aslam et al. 2020) Let $X$ be a non-empty set. $\mathrm{A}$ NCS in $\mathrm{X}$ is a pair $\mathcal{A}=(\mathrm{A}, \Lambda)$, 
where $\mathrm{A}=\left\{<\mathrm{x},\left[\mathrm{A}_{\mathrm{T}}^{\mathrm{L}}(\mathrm{x}), \mathrm{A}_{\mathrm{T}}^{\mathrm{U}}(\mathrm{x})\right],\left[\mathrm{A}_{\mathrm{I}}^{\mathrm{L}}(\mathrm{x}), \mathrm{A}_{\mathrm{I}}^{\mathrm{U}}(\mathrm{x})\right],\left[\mathrm{A}_{\mathrm{F}}^{\mathrm{L}}\right.\right.$ $\left.\left.(\mathrm{x}), \mathrm{A}_{\mathrm{F}}^{\mathrm{U}}(\mathrm{x})\right]>\mid \mathrm{x} \in \mathrm{X}\right\}$ is an interval neutrosophic set in $\mathrm{X}$ and $\Lambda=\left\{<\mathrm{x}, \lambda_{\mathrm{T}}(\mathrm{x}), \lambda_{\mathrm{I}}(\mathrm{x}), \lambda_{\mathrm{F}}(\mathrm{x})>\mid \mathrm{x} \in \mathrm{X}\right\}$ is a single-valve neutrosophic set in $\mathrm{X}$.

For simplicity, a basic element $\left\{<\mathrm{x},\left[\mathrm{A}_{\mathrm{T}}^{\mathrm{L}}(\mathrm{x}), \mathrm{A}_{\mathrm{T}}^{\mathrm{U}}(\mathrm{x})\right]\right.$, $\left.\left[\mathrm{A}_{\mathrm{I}}^{\mathrm{L}}(\mathrm{x}), \mathrm{A}_{\mathrm{I}}^{\mathrm{U}}(\mathrm{x})\right],\left[\mathrm{A}_{\mathrm{F}}^{\mathrm{L}}(\mathrm{x}), \mathrm{A}_{\mathrm{F}}^{\mathrm{U}}(\mathrm{x})\right], \lambda_{\mathrm{T}}(\mathrm{x}), \lambda_{\mathrm{I}}(\mathrm{x}), \lambda_{\mathrm{F}}(\mathrm{x})>\right\}$ in a NCS can be expressed as a $=\left(<\left[\mathrm{A}_{\mathrm{T}}^{\mathrm{L}}, \mathrm{A}_{\mathrm{T}}^{\mathrm{U}}\right],\left[\mathrm{A}_{\mathrm{I}}^{\mathrm{L}}, \mathrm{A}_{\mathrm{I}}^{\mathrm{U}}\right]\right.$, $\left[\mathrm{A}_{\mathrm{F}}^{\mathrm{L}}, \mathrm{A}_{\mathrm{F}}^{\mathrm{U}}\right]>,\left\langle\lambda_{\mathrm{T}}, \lambda_{\mathrm{I}}, \lambda_{\mathrm{F}}>\right)$, which is called neutrosophic cubic number (NCN). Besides, $A_{T}^{L}, A_{T}^{U}$, $\mathrm{A}_{\mathrm{I}}^{\mathrm{L}}, \mathrm{A}_{\mathrm{I}}^{\mathrm{U}}, \mathrm{A}_{\mathrm{F}}^{\mathrm{L}}, \mathrm{A}_{\mathrm{F}}^{\mathrm{U}}, \lambda_{\mathrm{T}}, \lambda_{\mathrm{I}}$ and $\lambda_{\mathrm{F}} \in[0,1]$, and $0 \leq \mathrm{A}_{\mathrm{T}}^{\mathrm{U}}+\mathrm{A}$ ${ }_{\mathrm{I}}^{\mathrm{U}}+\mathrm{A}_{\mathrm{F}}^{\mathrm{U}} \leq 3,0 \leq \lambda_{\mathrm{T}}+\lambda_{\mathrm{I}}+\lambda_{\mathrm{F}} \leq 3$.

Definition 9. (Pramanik et al. 2017; Ali et al. 2016; Liu et al. 2020) For any two NCNs $\mathcal{A}=\left\{<\left[\mathrm{A}_{\mathrm{T}}^{\mathrm{L}}\right.\right.$, $\left.\left.\mathrm{A}_{\mathrm{T}}^{\mathrm{U}}\right],\left[\mathrm{A}_{\mathrm{I}}^{\mathrm{L}}, \mathrm{A}_{\mathrm{I}}^{\mathrm{U}}\right],\left[\mathrm{A}_{\mathrm{F}}^{\mathrm{L}}, \mathrm{A}_{\mathrm{F}}^{\mathrm{U}}\right]>,<\lambda_{\mathrm{T}}, \lambda_{\mathrm{I}}, \lambda_{\mathrm{F}}>\right\}$ and $\mathcal{B}=$ $\left\{<\left[\mathrm{B}_{\mathrm{T}}^{\mathrm{L}}, \mathrm{B}_{\mathrm{T}}^{\mathrm{U}}\right],\left[\mathrm{B}_{\mathrm{I}}^{\mathrm{L}}, \mathrm{B}_{\mathrm{I}}^{\mathrm{U}}\right],\left[\mathrm{B}_{\mathrm{F}}^{\mathrm{L}}, \mathrm{B}_{\mathrm{F}}^{\mathrm{U}}\right]>,<\lambda_{\mathrm{T}}{ }^{\prime}, \lambda_{\mathrm{I}}{ }^{\prime}, \lambda_{\mathrm{F}}{ }^{\prime}>\right\}$ $(\mathrm{i}=1,2, \ldots \mathrm{n})$ and $\eta \geq 0$, the operational laws for NCNs are shown as follows:

(1)

$$
\begin{gathered}
\mathcal{A} \oplus \mathcal{B}=\left\{\left\langle\left[A_{T}^{L}+B_{T}^{L}-A_{T}^{L} B_{T}^{L}, A_{T}^{U}+B_{T}^{U}-A_{T}^{U} B_{T}^{U}\right],\left[A_{I}^{L} B_{I}^{L}, A_{I}^{U} B_{I}^{U}\right],\right.\right. \\
\left.\left.\left[A_{F}^{L} B_{F}^{L}, A_{F}^{U} B_{F}^{U}\right]\right\rangle,\left\langle\lambda_{T}+\lambda_{T}^{\prime}-\lambda_{T} \lambda_{T}^{\prime}, \lambda_{I} \lambda_{I}^{\prime}, \lambda_{F} \lambda_{F}^{\prime}\right\rangle\right\} .
\end{gathered}
$$

$$
\begin{aligned}
\mathcal{A} \otimes \mathcal{B}= & \left\{\left\langle\left[A_{T}^{L} B_{T}^{L}, A_{T}^{U} B_{T}^{U}\right],\left[A_{I}^{L}+B_{I}^{L}-A_{I}^{L} B_{I}^{L}, A_{I}^{U}+B_{I}^{U}-A_{I}^{U} B_{I}^{U}\right],\right.\right. \\
& {\left.\left[A_{F}^{L}+B_{F}^{L}-A_{F}^{L} B_{F}^{L}, A_{F}^{U}+B_{F}^{U}-A_{F}^{U} B_{F}^{U}\right]\right\rangle,\left\langle\lambda_{T} \lambda_{T}^{\prime}, \lambda_{I}+\lambda_{I}^{\prime}\right.} \\
& \left.\left.-\lambda_{I} \lambda_{I}^{\prime}, \lambda_{F}+\lambda_{F}^{\prime}-\lambda_{F} \lambda_{F}^{\prime}\right\rangle\right\} .
\end{aligned}
$$

$$
\begin{gathered}
\eta \otimes \mathcal{A}=\left\{\left\langle\left[1-\left(1-A_{T}^{L}\right)^{\eta}, 1-\left(1-A_{T}^{U}\right)^{\eta}\right],\left[\left(A_{I}^{L}\right)^{\eta},\left(A_{I}^{U}\right)^{\eta}\right],\right.\right. \\
\left.\left.\left[\left(A_{F}^{L}\right)^{\eta},\left(A_{F}^{U}\right)^{\eta}\right]\right\rangle,\left\langle 1-\left(1-\lambda_{T}\right)^{\eta},\left(\lambda_{I}\right)^{\eta},\left(\lambda_{F}\right)^{\eta}\right\rangle\right\} .
\end{gathered}
$$

4)

$$
\begin{aligned}
\mathcal{A}^{\eta}= & \left\{\left\langle\left[\left(A_{T}^{L}\right)^{\eta},\left(A_{T}^{U}\right)^{\eta}\right],\left[1-\left(1-A_{I}^{L}\right)^{\eta}, 1-\left(1-A_{I}^{U}\right)^{\eta}\right],\left[1-\left(1-A_{F}^{L}\right)^{\eta},\right.\right.\right. \\
& \left.\left.\left.1-\left(1-A_{F}^{U}\right)^{\eta}\right]\right\rangle,\left\langle\left(\lambda_{T}\right)^{\eta}, 1-\left(1-\lambda_{I}\right)^{\eta}, 1-\left(1-\lambda_{F}\right)^{\eta}\right\rangle\right\} .
\end{aligned}
$$

Definition 10. (Ali et al. 2016) For any two NCSs $\mathcal{A}_{\mathrm{i}}=\left\{<\left[\mathrm{A}_{\mathrm{T}}^{\mathrm{L}}\left(\mathrm{x}_{\mathrm{i}}\right), \mathrm{A}_{\mathrm{T}}^{\mathrm{U}}\left(\mathrm{x}_{\mathrm{i}}\right)\right],\left[\mathrm{A}_{\mathrm{I}}^{\mathrm{L}}\left(\mathrm{x}_{\mathrm{i}}\right), \mathrm{A}_{\mathrm{I}}^{\mathrm{U}}\left(\mathrm{x}_{\mathrm{i}}\right)\right],\left[\mathrm{A}_{\mathrm{F}}^{\mathrm{L}}, \mathrm{A}_{\mathrm{F}}^{\mathrm{U}}\right]>\right.$, $\left.<\lambda_{\mathrm{T}}\left(\mathrm{x}_{\mathrm{i}}\right), \lambda_{\mathrm{I}}\left(\mathrm{x}_{\mathrm{i}}\right), \lambda_{\mathrm{F}}\left(\mathrm{x}_{\mathrm{i}}\right)>\right\}$ and $\mathcal{B}_{\mathrm{i}}=\left\{<\left[\mathrm{B}_{\mathrm{T}}^{\mathrm{L}}\left(\mathrm{x}_{\mathrm{i}}\right), \mathrm{B}_{\mathrm{T}}^{\mathrm{U}}\left(\mathrm{x}_{\mathrm{i}}\right)\right]\right.$, $\left[\mathrm{B}_{\mathrm{I}}^{\mathrm{L}}\left(\mathrm{x}_{\mathrm{i}}\right), \mathrm{B}_{\mathrm{I}}^{\mathrm{U}}\left(\mathrm{x}_{\mathrm{i}}\right)\right],\left[\mathrm{B}_{\mathrm{F}}^{\mathrm{L}}\left(\mathrm{x}_{\mathrm{i}}\right), \mathrm{B}_{\mathrm{F}}^{\mathrm{U}}\left(\mathrm{x}_{\mathrm{i}}\right)\right]>,<\lambda_{\mathrm{T}}{ }^{\prime}\left(\mathrm{x}_{\mathrm{i}}\right), \lambda_{\mathrm{I}}{ }^{\prime}\left(\mathrm{x}_{\mathrm{i}}\right)$, $\left.\lambda_{\mathrm{F}}{ }^{\prime}\left(\mathrm{x}_{\mathrm{i}}\right)>\right\} \quad(\mathrm{i}=1,2, \ldots \mathrm{n})$, the Hamming distance measure between $\mathcal{A}$ and $\mathcal{B}$ is given by:

$$
\begin{aligned}
d(\mathcal{A}, \mathcal{B})= & \frac{1}{9 n} \sum_{i=1}^{n}\left(\left|A_{T}^{L}\left(x_{i}\right)-B_{T}^{L}\left(x_{i}\right)\right|+\left|A_{T}^{U}\left(x_{i}\right)-B_{T}^{U}\left(x_{i}\right)\right|\right. \\
& +\left|A_{I}^{L}\left(x_{i}\right)-B_{I}^{L}\left(x_{i}\right)\right|+\left|A_{I}^{U}\left(x_{i}\right)-B_{I}^{U}\left(x_{i}\right)\right| \\
& +\left|A_{F}^{L}\left(x_{i}\right)-B_{F}^{L}\left(x_{i}\right)\right|+\left|A_{F}^{U}\left(x_{i}\right)-B_{F}^{U}\left(x_{i}\right)\right| \\
& \left.+\left|\lambda_{T}\left(x_{i}\right)-\lambda_{T}^{(}\left(x_{i}\right)\right|+\left|\lambda_{I}\left(x_{i}\right)-\lambda_{I}^{\prime}\left(x_{i}\right)\right|+\left|\lambda_{F}\left(x_{i}\right)-\lambda_{F}^{\prime}\left(x_{i}\right)\right|\right) .
\end{aligned}
$$

Definition 11. (Ye 2018) Let $\mathcal{A}_{1}, \mathcal{A}_{2}, \ldots, \mathcal{A}_{n}$ be a set of NCNs where $\mathcal{A}_{\mathrm{j}}=\left\{<\left[\mathrm{A}_{\mathrm{Tj}}^{\mathrm{L}}, \mathrm{A}_{\mathrm{Tj}}^{\mathrm{U}}\right],\left[\mathrm{A}_{\mathrm{lj}}^{\mathrm{L}}, \mathrm{A}_{\mathrm{Ij}}^{\mathrm{U}}\right]\right.$, $\left[\mathrm{A}_{\mathrm{Fj}}^{\mathrm{L}}, \mathrm{A}_{\mathrm{Fj}}^{\mathrm{U}}\right]>,\left\langle\lambda_{\mathrm{Tj}}, \lambda_{\mathrm{Ij}}, \lambda_{\mathrm{Fj}}>\right\}$. Then the neutrosophic cubic weighted arithmetic averaging (NCWAA) operator is defined as

$$
\begin{aligned}
& N C W A A\left(a_{1}, a_{2}, \ldots a_{n}\right) \\
= & \sum_{j=1}^{n} w_{j} a_{j} \\
= & \left\{\left\langle\left[1-\prod_{j=1}^{n}\left(1-A_{T_{j}}^{L}\right)^{w_{j}}, 1-\prod_{j=1}^{n}\left(1-A_{T_{j}}^{U}\right)^{w_{j}}\right],\right.\right. \\
& {\left.\left[\prod_{j=1}^{n}\left(A_{I_{j}}^{L}\right)^{w_{j}}, \prod_{j=1}^{n}\left(A_{I_{j}}^{U}\right)^{w_{j}}\right],\left[\prod_{j=1}^{n}\left(A_{F_{j}}^{L}\right)^{w_{j}}, \prod_{j=1}^{n}\left(A_{F_{j}}^{U}\right)^{w_{j}}\right]\right\rangle, } \\
& \left.\left\langle 1-\prod_{j=1}^{n}\left(1-\lambda_{T_{j}}\right)^{w_{j}}, \prod_{j=1}^{n}\left(\lambda_{I_{j}}\right)^{w_{j}}, \prod_{j=1}^{n}\left(\lambda_{F_{j}}\right)^{w_{j}}\right\rangle\right\}
\end{aligned}
$$

When $\mathrm{w}_{\mathrm{j}}=1 / \mathrm{n}$, the NCWAA operator degenerates to NCAA operator, i.e.

$\operatorname{NCAA}\left(a_{1}, a_{2}, \ldots a_{n}\right)$

$$
=\frac{1}{n} \sum_{j=1}^{n} a_{j}
$$$$
=\left\{\left\langle\left[1-\prod_{j=1}^{n}\left(1-A_{T_{j}}^{L}\right)^{\frac{1}{n}}, 1-\prod_{j=1}^{n}\left(1-A_{T_{j}}^{U}\right)^{\frac{1}{n}}\right]\right.\right. \text {, }
$$$$
\left.\left[\prod_{j=1}^{n}\left(A_{I_{j}}^{L}\right)^{\frac{1}{n}}, \prod_{j=1}^{n}\left(A_{I_{j}}^{U}\right)^{\frac{1}{n}}\right],\left[\prod_{j=1}^{n}\left(A_{F_{j}}^{L}\right)^{\frac{1}{n}}, \prod_{j=1}^{n}\left(A_{F_{j}}^{U}\right)^{\frac{1}{n}}\right]\right\rangle,
$$$$
\left.\left\langle 1-\prod_{j=1}^{n}\left(1-\lambda_{T_{j}}\right)^{\frac{1}{n}}, \prod_{j=1}^{n}\left(\lambda_{I_{j}}\right)^{\frac{1}{n}}, \prod_{j=1}^{n}\left(\lambda_{F_{j}}\right)^{\frac{1}{n}}\right\rangle\right\}
$$

The Maclaurin symmetric mean (MSM) operator (Maclaurin 1986)was initiated by Maclaurin originally, which can seize the interrelation among attributes more effectively.

Definition 12. Suppose $a_{i}(i=1,2, \ldots n)$ is a set of non-negative crisp numbers, then the Maclaurin symmetric mean (MSM) operator is the mapping shown as: 


$$
\operatorname{MSM}^{(k)}\left(a_{1}, a_{2}, \ldots a_{n}\right)=\left(\frac{\sum_{1 \leq i_{1} \leq \ldots i_{k} \leq n} \prod_{j=1}^{k} a_{i_{j}}}{C_{n}^{k}}\right)^{1 / k},
$$

where $\mathrm{k}=1,2, \ldots, \mathrm{n},\left(\mathrm{i}_{1}, \mathrm{i}_{2}, \ldots, \mathrm{i}_{\mathrm{k}}\right)$ is one of all the $\mathrm{k}$ tuple combinations of $(1,2, \ldots \mathrm{n})$, and $\mathrm{C}_{\mathrm{n}}^{\mathrm{k}}$ is the binomial coefficient which satisfies:

$$
C_{n}^{k}=\frac{n !}{k !(n-k) !} \text {. }
$$

Definition 13. (Qin 2015) Suppose $a_{i}(i=1,2, \ldots n)$ is a set of non-negative crisp numbers, then the dual Maclaurin symmetric mean (DMSM) operator is the mapping shown as:

$$
\operatorname{DMSM}^{(k)}\left(a_{1}, a_{2}, \ldots a_{n}\right)=\frac{1}{k}\left(\prod_{1 \leq i_{1}<\ldots<i_{k} \leq n}\left(\sum_{j=1}^{k} a_{i_{j}}\right)^{\frac{1}{c_{c}^{k}}}\right),
$$

where $k=1,2, \ldots, n,\left(i_{1}, i_{2}, \ldots, i_{k}\right)$ is one of all the $k$ tuple combinations of $(1,2, \ldots n)$, and $\mathrm{C}_{n}{ }_{n}$ is the binomial coefficient satisfying following formula:

$$
C_{n}^{k}=\frac{n !}{k !(n-k) !} \text {. }
$$

Definition 14. The MSM operator possesses some characteristics:

(1) Idempotency: $\operatorname{MSM}^{(k)}\left(a_{1}, a_{2}, \ldots a_{n}\right)=a$ when $a_{i}$ $=\mathrm{a}(\mathrm{i}=1,2, \ldots \mathrm{n})$;

(2) Monotonicity: $\operatorname{MSM}^{(\mathrm{k})}\left(\mathrm{a}_{1}, \mathrm{a}_{2}, \ldots \mathrm{a}_{\mathrm{n}}\right) \leq \operatorname{MSM}^{(\mathrm{k})}$ $\left(b_{1}, b_{2}, \ldots b_{n}\right)$ if $a_{i} \leq b_{i}(i=1,2, \ldots n)$;

(3) Boundedness: $\min \left\{a_{i}\right\} \leq \operatorname{MSM}^{(k)}\left(a_{1}, a_{2}, \ldots a_{n}\right)$ $\leq \max \left\{\mathrm{a}_{\mathrm{i}}\right\}$.

Definition 15. (Bai 2018; Nguyen 2020; Liu 2020) Let $A=\left(a_{1}, a_{2}, \ldots a_{n}\right)$ be a set of non-negative real numbers, which are divided into $d$ partitions $Z_{1}, Z_{2}, \ldots$ $Z_{d}$ and $Z_{i} \cap Z_{j}=\emptyset$. Then the PMSM operator is defined as follows:

$$
\operatorname{PMSM}^{\left(k_{1}, k_{2}, \ldots k_{d}\right)}\left(a_{1}, a_{2}, \ldots, a_{n}\right)=\frac{1}{d} \sum_{r=1}^{d}\left(\frac{\sum_{1 \leqslant i_{1}<\ldots i_{r} \leq \leq z r \mid}\left(\prod_{j=1}^{k r} a_{i_{j}}\right)}{C_{|Z r|}^{k r}}\right)^{1 / k r},
$$

where $k_{r}$ is the parameter in the partition $Z_{r}, k=1$, $2, \ldots,\left|Z_{r}\right| \cdot\left|Z_{r}\right|$ is the number of input arguments in the partition $Z_{r}$ and it satisfies $\sum_{r=1}^{d}|Z r|=n .\left(i_{1}, i_{2}, \ldots, i_{k}\right)$ traverses the overall $\mathrm{k}_{\mathrm{r}}$-tuple different combinations of $\left(1,2, \ldots,\left|Z_{r}\right|\right) ; C_{|Z r|}^{k r}$ is the binomial coefficient and it satisfies:

$$
C_{|Z r|}^{k r}=\frac{\left|Z_{r}\right| !}{k_{r} !\left(\left|Z_{r}\right|-k_{r}\right) !} .
$$

Definition 16. (Liu 2020; Liu 2020) Let $A=\left\{a_{1}\right.$, $\left.a_{2}, \ldots a_{n}\right\}$ be a set of non-negative real numbers, which are divided into $d$ partitions $Z_{1}, Z_{2}, \ldots Z_{d}$ and $Z_{i} \cap Z_{j}=\emptyset$. The weight vector of input arguments is $\mathrm{W}=\left(\mathrm{w}_{1}, \mathrm{w}_{2}, \ldots, \mathrm{w}_{\mathrm{n}}\right)^{\mathrm{T}}$ with $\mathrm{w}_{\mathrm{i}} \in[0$, 1] and $\sum_{i}^{n} \mathrm{w}_{i}=1$. Then the weighted partitioned Maclaurin symmetric mean (WPMSM) operator is defined as follows:

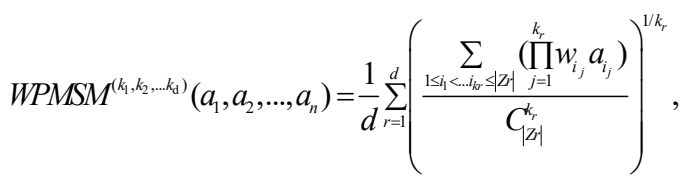

where $\mathrm{k}_{\mathrm{r}}$ is the parameter in the partition $\mathrm{Z}_{\mathrm{r}}, \mathrm{k}=1$, $2, \ldots,\left|Z_{r}\right| \cdot\left|Z_{r}\right|$ is the number of input arguments in the partition $Z_{r}$ and it satisfies $\sum_{r=1}^{d}|Z r|=n$. (i , $i_{2}, \ldots, i_{k}$ ) traverses the overall $k_{r}$ - tuple different combinations of $\left(1,2, \ldots,\left|Z_{r}\right|\right) ; C_{|\mathrm{Zr}|}^{\mathrm{kr}}$ is the binomial coefficient and it satisfies :

$$
C_{|Z r|}^{k r}=\frac{\left|Z_{r}\right| !}{k_{r} !\left(\left|Z_{r}\right|-k_{r}\right) !} \text {. }
$$

\section{The NCPMSM operator and NCWPMSM operator and a novel score function of NCN}

In this part, firstly we apply the PMSM operator to neutrosophic cubic environment and propose the NCPMSM operator and NCWPMSM operator.

Definition 17. Let $\mathcal{A}_{1}, \mathcal{A}_{2}, \ldots, \mathcal{A}_{n}$ be a set of NCNs where $\mathcal{A}_{\mathrm{i}}=\left\{<\left[\mathrm{A}_{\mathrm{Ti}}^{\mathrm{L}}, \mathrm{A}_{\mathrm{Ti}}^{\mathrm{U}}\right],\left[\mathrm{A}_{\mathrm{Ii}}^{\mathrm{L}}, \mathrm{A}_{\mathrm{Ii}}^{\mathrm{U}}\right],\left[\mathrm{A}_{\mathrm{Fi}}^{\mathrm{L}}, \mathrm{A}\right.\right.$ $\left.{ }_{\mathrm{Fi}}^{\mathrm{U}}\right]>,\left\langle\lambda_{\mathrm{Ti}}, \lambda_{\mathrm{Ii}}, \lambda_{\mathrm{Fi}}>\right\}$. Then the NCPMSM operator is defined as follows:

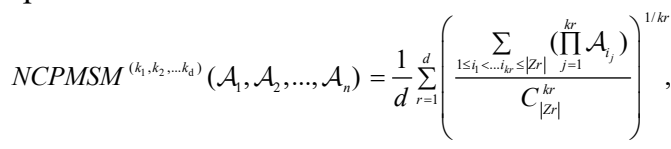

where $\mathrm{k}_{\mathrm{r}}$ is the parameter in the partition $\mathrm{Z}_{\mathrm{r}}, \mathrm{k}=1$, $2, \ldots,\left|Z_{r}\right| .\left|Z_{r}\right|$ indicates the number of input 
arguments in the partition $\mathrm{Z}_{\mathrm{r}}$ and $\sum_{\mathrm{r}=1}^{\mathrm{d}}|\mathrm{Zr}|=\mathrm{n}$. ( $\mathrm{i}_{1}$, $\left.\mathrm{i}_{2}, \ldots, \mathrm{i}_{\mathrm{k}}\right)$ traverses the overall $\mathrm{k}_{\mathrm{r}}$-tuple different combinations of $\left(1,2, \ldots,\left|\mathrm{Z}_{\mathrm{r}}\right|\right) ; \mathrm{C}_{|\mathrm{Zr}|}^{\mathrm{kr}}$ is the binomial coefficient and it satisfies:

$$
C_{|z r|}^{k r}=\frac{n !}{k_{r} !\left(\mathrm{n}-\left|Z_{r}\right|\right) !} .
$$

Theorem 1. Let $\mathcal{A}_{1}, \mathcal{A}_{2}, \ldots \mathcal{A}_{n}$ be a set of NCNs. Then the integrated result of the above NCPMSM operator is also a NCN, which displayed as below:

$$
\begin{aligned}
& \operatorname{NCPMSM}^{\left(k_{1}, k_{2}, \ldots k_{\mathrm{d}}\right)}\left(\mathcal{A}_{1}, \mathcal{A}_{2}, \ldots \mathcal{A}_{n}\right) \\
& =\frac{1}{d} \sum_{r=1}^{d}\left(\frac{\sum_{1 \leqslant i_{1}<\ldots, i_{r} \leq\left|z_{r}\right|}\left(\prod_{j=1}^{k_{r}} \mathcal{A}_{i_{j}}\right)}{C_{\left|Z r_{r}\right|}^{k_{r}}}\right)^{1 / k_{r}} \\
& =\left\{\left\langle\left[1-\prod_{r=1}^{d}\left(1-\left(1-\prod_{1 \leq i_{1}<\ldots i_{r r} \leq|Z r|}\left(1-\prod_{j=1}^{k_{r}} A_{T_{i_{j}}}^{L}\right)^{1 /\left.\right|_{|\not r|} ^{k_{r}}}\right)^{1 / k_{r}}\right)^{\frac{1}{d}},\right.\right.\right. \\
& \left.1-\prod_{r=1}^{d}\left(1-\left(1-\prod_{1 \leq i_{1}<\ldots i_{r} \leq \leq z r \mid}\left(1-\prod_{j=1}^{k_{r}} A_{T_{i_{j}}^{U}}^{U}\right)^{1 / C_{|r r|}^{k_{r} \mid}}\right)^{1 / k_{r}}\right)^{\frac{1}{d}}\right], \\
& {\left[\prod_{r=1}^{d}\left(1-\left(1-\prod_{1 \leq i_{1}<\ldots i_{r_{r}} \leq\left|z r_{r}\right|}\left(1-\prod_{j=1}^{k_{r}}\left(1-A_{I_{i_{j}}}^{L}\right)\right)^{1 / C_{\mid z_{r}-1}^{k_{r}}}\right)^{1 / k_{r}}\right)^{\frac{1}{d}},\right.} \\
& \left.\prod_{r=1}^{d}\left(1-\left(1-\prod_{1 \leq i_{1}<\ldots i_{k_{r}} \leq\left|z r_{r}\right|}\left(1-\prod_{j=1}^{k_{r}}\left(1-A_{t_{i_{j}}}^{U}\right)\right)^{1 / C_{\left|r_{r \mid}\right|}^{k_{r}}}\right)^{1 / k_{r}}\right)^{\frac{1}{d}}\right], \\
& {\left[\prod_{r=1}^{d}\left(1-\left(1-\prod_{1 \leq i_{1}<\ldots, i_{k r} \leq|z r|}\left(1-\prod_{j=1}^{k_{r}}\left(1-A_{F_{i_{j}}}^{L}\right)\right)^{1 / C_{\mid r_{r}}^{k_{r}}}\right)^{1 / k_{r}}\right)^{\frac{1}{d}},\right.} \\
& \left.\left.\prod_{r=1}^{d}\left(1-\left(1-\prod_{1 \leq i_{1} \leqslant \ldots i_{k r} \leq\left|z r_{r}\right|}\left(1-\prod_{j=1}^{k_{r}}\left(1-A_{F_{i_{j}}}^{U}\right)\right)^{1 / \mathcal{C}_{\left|r_{r}\right|}^{k_{r}}}\right)^{1 / k_{r}}\right)^{\frac{1}{d}}\right]\right\rangle,
\end{aligned}
$$

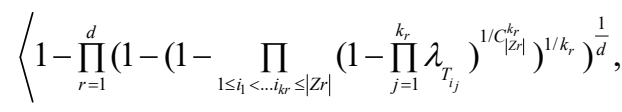

$$
\begin{aligned}
& \prod_{r=1}^{d}\left(1-\left(1-\prod_{\leq i_{i}<\ldots, i_{r r} \leq|z r|}\left(1-\prod_{j=1}^{k_{r}}\left(1-\lambda_{I_{i_{j}}}\right)\right)^{1 /\left.\right|_{\left|r_{r}\right|} ^{k_{r} \mid}}\right)^{1 / k_{r}}\right)^{\frac{1}{d}},
\end{aligned}
$$

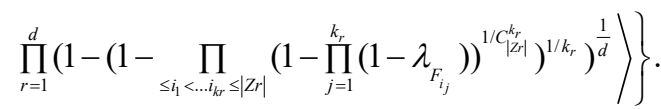

Proof. According to Definition 9, we have $\prod_{j=1}^{k_{r}} \mathcal{A}_{i_{j}}$ $=\left\{\left\langle\left[\prod_{j=1}^{k_{r}} A_{T_{i_{j}}}^{L}, \prod_{j=1}^{k_{r}} A_{T_{i_{j}}}^{U}\right],\left[1-\prod_{j=1}^{k_{r}}\left(1-A_{I_{i_{j}}}^{L}\right), 1-\prod_{j=1}^{k_{r}}\left(1-A_{I_{i_{j}}}^{U}\right)\right]\right.\right.$,

$$
\begin{aligned}
& \left.\left[1-\prod_{j=1}^{k_{r}}\left(1-A_{F_{i_{j}}}^{L}\right), 1-\prod_{j=1}^{k_{r}}\left(1-A_{F_{i_{j}}}^{U}\right)\right]\right\rangle, \\
& \left.\left\langle\prod_{j=1}^{k_{r}} \lambda_{T_{i_{j}}}, 1-\prod_{j=1}^{k_{r}}\left(1-\lambda_{I_{i_{j}}}\right), 1-\prod_{j=1}^{k_{r}}\left(1-\lambda_{F_{i j}}\right)\right\rangle\right\},
\end{aligned}
$$

So,

$$
\begin{aligned}
& \sum_{1 \leq i_{1}<\ldots i_{k_{r}} \leq\left|z r_{r}\right|}\left(\prod_{j=1}^{k_{r}} \mathcal{A}_{i_{j}}\right) \\
& =\left\{\left\langle\left[1-\prod_{1 \leq i_{i}<\ldots i_{k} \leq|z r r|}\left(1-\prod_{j=1}^{k_{r}} A_{T_{i_{j}}}^{L}\right), 1-\prod_{1 \leq i_{i}<\ldots i_{k_{r}} \leq \gamma_{r} \mid}\left(1-\prod_{j=1}^{k_{r}} A_{T_{i_{j}}}^{U}\right)\right],\right.\right.
\end{aligned}
$$

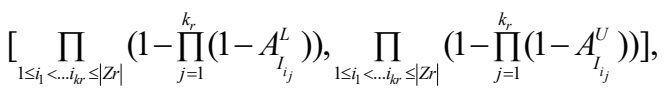

$$
\begin{aligned}
& \left.\left[\prod_{1 \leq \zeta_{i}<\ldots i_{r r} \leq\left|Z_{i}\right|}\left(1-\prod_{j=1}^{k_{r}}\left(1-A_{F_{i_{j}}}^{L}\right)\right), \prod_{1 \leq i_{i}<\ldots i_{r} \leq \leq Z_{r} \mid}\left(1-\prod_{j=1}^{k_{r}}\left(1-A_{F_{i_{j}}}^{U}\right)\right)\right]\right\rangle \text {, }
\end{aligned}
$$

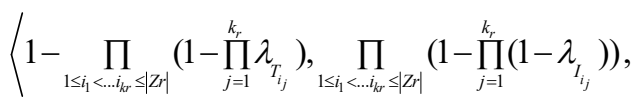

$$
\begin{aligned}
& \left.\left.\prod_{1 \leq i_{i}<\ldots i_{r} \leq \leq r_{i} \mid}\left(1-\prod_{j=1}^{k_{r}}\left(1-\lambda_{F_{i_{j}}}\right)\right)\right\rangle\right\} \text {. }
\end{aligned}
$$

Then there are further aggregated results:

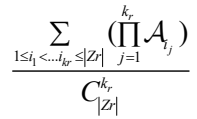

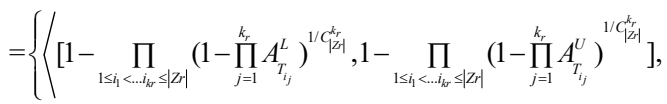

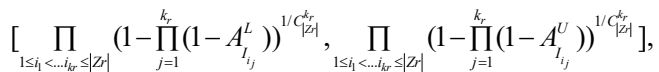

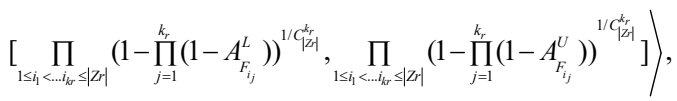

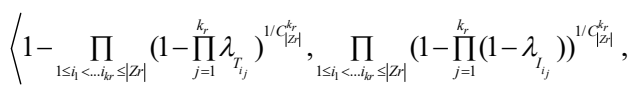

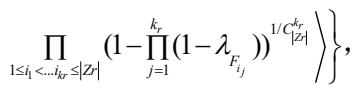

Further,

$$
\begin{aligned}
& \left(\frac{\sum_{1 \leq i_{1}<\ldots i_{r r} \leq|z r|}\left(\prod_{j=1}^{k_{r}} \mathcal{A}_{i_{j}}\right)}{C_{|z r|}^{k_{r} \mid}}\right)^{1 / k_{r}} \\
& =\left\{\left\langle\left[\left(1-\prod_{1 \leq i_{i}<\ldots i_{k_{r}} \leq|z r|}\left(1-\prod_{j=1}^{k_{r}} A_{T_{i_{j}}}^{L}\right)^{1 / c_{|z r|}^{k_{r}}}\right)^{1 / k_{r}},\right.\right.\right. \\
& \left.\left(1-\prod_{1 \leq i_{i}<\ldots i_{k_{r}} \leq|z r|}\left(1-\prod_{j=1}^{k_{r}} A_{T_{i_{j}}}^{U}\right)^{1 / C_{|2 r|}^{k_{r}}}\right)^{1 / k_{r}}\right],
\end{aligned}
$$




$$
\begin{aligned}
& {\left[1-\left(1-\prod_{1 \leq i_{1} \leq \ldots i_{r r} \leq|Z| r \mid}\left(1-\prod_{j=1}^{k_{r}}\left(1-A_{I_{i_{j}}}^{L}\right)\right)^{1 / C_{|2|}^{k_{r}}}\right)^{1 / k_{r}},\right.} \\
& \left.1-\left(1-\prod_{1 \leq i_{1}<\ldots, i_{r} \leq|z r|}\left(1-\prod_{j=1}^{k_{r}}\left(1-A_{I_{i_{j}}}^{U}\right)\right)^{1 / C_{\mid\langle-|r|}^{k_{r}}}\right)^{1 / k_{r}}\right] \text {, }
\end{aligned}
$$

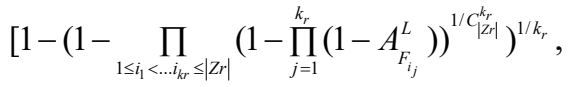

$$
\begin{aligned}
& \left.\left.1-\left(1-\prod_{1 \leq \zeta_{i}<\ldots i_{r} \leq\left|z r_{r}\right|}\left(1-\prod_{j=1}^{k_{r}}\left(1-A_{F_{i j}}^{U}\right)\right)^{1 /\left.\right|_{\left|k_{r}\right|} ^{k_{r}}}\right)^{1 / k_{r}}\right]\right\rangle, \\
& \left\langle\left(1-\prod_{1 \leq i_{1}<\ldots i_{r r} \leq|z r|}\left(1-\prod_{j=1}^{k_{r}} \lambda_{T_{i_{j}}}\right)^{1 / c_{|z r|}^{k_{r}}}\right)^{1 / k_{r}},\right. \\
& 1-\left(1-\prod_{1 \leq i_{i}<\ldots, i_{r r} \leq|z r|}\left(1-\prod_{j=1}^{k_{r}}\left(1-\lambda_{I_{i_{j}}}\right)\right)^{1 / c_{\mid \nu_{r}}^{k_{r}}}\right)^{1 / k_{r}}, \\
& \left.\left.1-\left(1-\prod_{1 \leq i_{i}<\ldots i_{r} \leq\left|z r_{r}\right|}\left(1-\prod_{j=1}^{k_{r}}\left(1-\lambda_{F_{i_{j}}}\right)\right)^{1 / C_{\mid z-r_{1}}^{k_{r}}}\right)^{1 / k_{r}}\right\rangle\right\},
\end{aligned}
$$

So,

$$
\begin{aligned}
& \sum_{r=1}^{d}\left(\frac{\sum_{1 \leq i_{1}<\ldots i_{k r} \leq|z r|}\left(\prod_{j=1}^{k_{r}} \mathcal{A}_{i_{j}}\right)}{C_{\left|Z r_{r}\right|}^{k_{r}}}\right)^{1 / k_{r}} \\
& =\left\{\left\langle\left[1-\prod_{r=1}^{d}\left(1-\left(1-\prod_{1 \leq i_{1}<\ldots, l_{l_{r}} \leq\left\langle z_{r}\right|}\left(1-\prod_{j=1}^{k_{r}} A_{T_{i_{j}}}^{L}\right)^{1 / C_{\left|r_{r}\right|}^{k_{r} \mid}}\right)^{1 / k_{r}}\right),\right.\right.\right. \\
& \left.1-\prod_{r=1}^{d}\left(1-\left(1-\prod_{1 \leq i_{1}<\ldots i_{k r} \leq\{z r \mid}\left(1-\prod_{j=1}^{k_{r}} A_{T_{i j}}^{U}\right)^{1 /\left.\right|_{|>r|} ^{k_{r}}}\right)^{1 / k_{r}}\right)\right], \\
& {\left[\prod_{r=1}^{d}\left(1-\left(1-\prod_{1 \leq i_{1}<\ldots, i_{k r} \leq\left|\not r_{r}\right|}\left(1-\prod_{j=1}^{k_{r}}\left(1-A_{I_{i_{j}}}^{L}\right)\right)^{1 / k_{\mid>-1}^{k_{r}}}\right)^{1 / k_{r}}\right),\right.} \\
& \left.\prod_{r=1}^{d}\left(1-\left(1-\prod_{1 \leq i_{1}<\ldots i_{r r} \leq\{r r \mid}\left(1-\prod_{j=1}^{k_{r}}\left(1-A_{I_{i_{j}}}^{U}\right)\right)^{1 / C_{|2|}^{k_{r}}}\right)^{1 / k_{r}}\right)\right], \\
& {\left[\prod_{r=1}^{d}\left(1-\left(1-\prod_{1 \leq i_{1}<\ldots i_{r r} \leq\left|\forall r_{r}\right|}\left(1-\prod_{j=1}^{k_{r}}\left(1-A_{F_{i_{j}}^{L}}^{L}\right)\right)^{1 / C_{\mid r_{n}}^{k_{r}}}\right)^{1 / k_{r}}\right),\right.}
\end{aligned}
$$

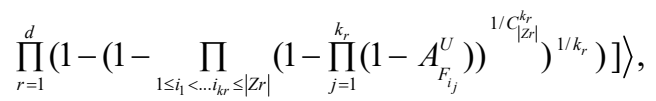

$$
\begin{aligned}
& \left\langle 1-\prod_{r=1}^{d}\left(1-\left(1-\prod_{1 \leq i_{1}<\ldots i_{k r} \leq\langle z r|}\left(1-\prod_{j=1}^{k_{r}} \lambda_{T_{i_{j}}}\right)^{1 / k_{|z|}^{k_{r}}}\right)^{1 / k_{r}}\right),\right. \\
& \prod_{r=1}^{d}\left(1-\left(1-\prod_{1 \leq i_{1}<\ldots i_{k r} \leq\left|r_{r}\right|}\left(1-\prod_{j=1}^{k_{r}}\left(1-\lambda_{I_{i_{j}}}\right)\right)^{1 / C_{|>-r|}^{k_{r}}}\right)^{1 / k_{r}}\right),
\end{aligned}
$$

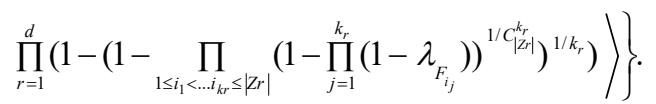

Finally, we obtain the aggregated value of $\mathrm{n}$ NCNs as follows:

$$
\begin{aligned}
& \operatorname{NCPMSM}^{\left(k_{1}, k_{2}, \ldots k_{\mathrm{d}}\right)}\left(\mathcal{A}_{1}, \mathcal{A}_{2}, \ldots, \mathcal{A}_{n}\right) \\
& =\frac{1}{d} \sum_{r=1}^{d}\left(\frac{\sum_{1 \leq i_{1}<\ldots i_{r r} \leq \leq z r \mid}\left(\prod_{j=1}^{k_{r}} \mathcal{A}_{i_{j}}\right)}{C_{\left|Z r_{r}\right|}^{k_{r}}}\right)^{1 / k_{r}} \\
& =\left\{\left\langle\left[1-\prod_{r=1}^{d}\left(1-\left(1-\prod_{1 \leq i_{1}<\ldots, i_{l_{r}} \leq|z r|}\left(1-\prod_{j=1}^{k_{r}} A_{T_{i_{j}}}^{L}\right)^{1 /\left.\right|_{\left|z_{r}\right|} ^{k_{r}}}\right)^{1 / k_{r}}\right)^{\frac{1}{d}},\right.\right.\right. \\
& \left.1-\prod_{r=1}^{d}\left(1-\left(1-\prod_{1 \leq i_{1}<\ldots i_{k r} \leq|Z r|}\left(1-\prod_{j=1}^{k_{r}} A_{T_{i_{j}}}^{U}\right)^{1 / C_{\left|r_{r}\right|}^{k_{r} \mid}}\right)^{1 / k_{r}}\right)^{\frac{1}{d}}\right],
\end{aligned}
$$

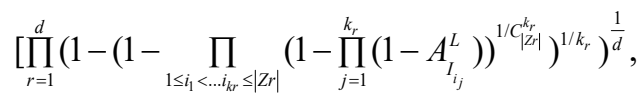

$$
\begin{aligned}
& \left.\prod_{r=1}^{d}\left(1-\left(1-\prod_{1 \leq i_{i}<\ldots, i_{r} \leq|z r|}\left(1-\prod_{j=1}^{k_{r}}\left(1-A_{I_{i_{j}}}^{U}\right)\right)^{1 / / k_{|r|}^{k_{r}}}\right)^{1 / k_{r}}\right)^{\frac{1}{d}}\right], \\
& {\left[\prod_{r=1}^{d}\left(1-\left(1-\prod_{1 \leq i_{1}<\ldots i_{k r} \leq|z r|}\left(1-\prod_{j=1}^{k_{r}}\left(1-A_{F_{i_{j}}}^{L}\right)\right)^{1 / C_{\left|r_{r}\right|}^{k_{r}}}\right)^{1 / k_{r}}\right)^{\frac{1}{d}},\right.} \\
& \left.\left.\prod_{r=1}^{d}\left(1-\left(1-\prod_{1 \leq i_{1}<\ldots i_{k_{r}} \leq\left|z r_{r}\right|}\left(1-\prod_{j=1}^{k_{r}}\left(1-A_{F_{i_{j}}}^{U}\right)\right)^{1 / \mathcal{C}_{\mid k_{r}}^{k_{r}}}\right)^{1 / k_{r}}\right)^{\frac{1}{d}}\right]\right\rangle,
\end{aligned}
$$

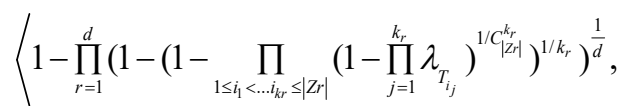

$$
\begin{aligned}
& \prod_{r=1}^{d}\left(1-\left(1-\prod_{1 \leq i_{1}<\ldots i_{r} \leq \leq z r \mid}\left(1-\prod_{j=1}^{k_{r}}\left(1-\lambda_{I_{i_{j}}}\right)\right)^{1 / k_{|2|}^{k_{r}}}\right)^{1 / k_{r}}\right)^{\frac{1}{d}},
\end{aligned}
$$

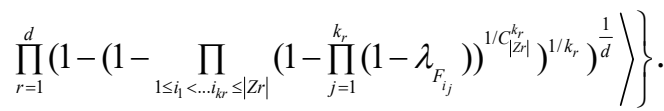

Next we prove some properties of the proposed NCPMSM operator.

Theorem 2. (commutativity) Let $\left(\mathcal{A}_{1}, \mathcal{A}_{2}, \ldots, \mathcal{A}_{n}\right)$ be any permutation of $\mathcal{A}_{1}, \mathcal{A}_{2}, \ldots, \mathcal{A}_{n}$; then $N C P M S M^{\left(k_{1}, k_{2}, \ldots k_{d}\right)}\left(\mathcal{A}, \mathcal{A}_{2}, \ldots, \mathcal{A}_{n}\right)=N C P M S M^{\left(k_{1}, k_{2}, \ldots k_{d}\right)}\left(\mathcal{A}_{1}^{\prime}\right.$, $\left.\mathcal{A}_{2}^{\prime}, \ldots, \mathcal{A}_{n}^{\prime}\right)$.

Proof. Let

$$
\begin{aligned}
& \operatorname{NCPMSM} M^{\left(k_{1}, k_{2}, \ldots k_{d}\right)}\left(\mathcal{A}_{1}, \mathcal{A}_{2}, \ldots \mathcal{A}_{n}\right) \\
& =\frac{1}{d} \sum_{r=1}^{d}\left(\frac{\sum_{1 \leq i_{1}<\ldots i_{k_{r}} \leq|z r|}\left(\prod_{j=1}^{k_{r}} \mathcal{A}_{i_{j}}\right)}{C_{|z r|}^{k_{r} \mid}}\right)^{1 / k_{r}}
\end{aligned}
$$

and 


$$
\begin{aligned}
& \operatorname{NCPMSM}^{\left(k_{1}, k_{2}, \ldots k_{\mathrm{d}}\right)}\left(\mathcal{A}_{1}^{\prime}, \mathcal{A}_{2}^{\prime}, \ldots \mathcal{A}_{n}^{\prime}\right) \\
& =\frac{1}{d} \sum_{r=1}^{d}\left(\frac{\sum_{1 \leq i_{1}<\ldots i_{r} \leq \leq|z|}\left(\prod_{j=1}^{k_{r}} \mathcal{A}_{i_{j}}\right)}{C_{\left|Z_{r}\right|}^{k_{r}}}\right)^{1 / k_{r}}
\end{aligned}
$$

Since $\left(\mathcal{A}_{1}^{\prime}, \mathcal{A}_{2}^{\prime}, \ldots, \mathcal{A}_{n}^{\prime}\right)_{k}$ be any permutation of $\mathcal{A}_{1}, \mathcal{A}_{2}, \ldots, \mathcal{A}_{n}$, we have $\prod_{j=1}^{k_{r}} \mathcal{A}_{i_{j}}=\prod_{j=1}^{k_{r}} \mathcal{A}_{i_{j}}^{\prime}$ and

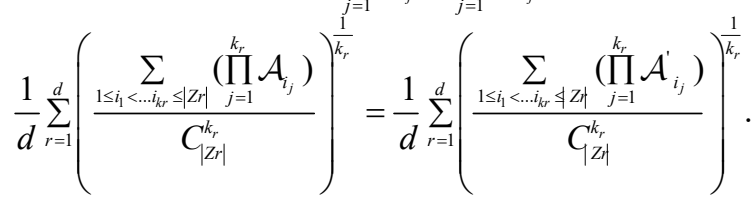

Such we can get $\operatorname{NCPMSM}^{\left(k_{1}, k_{2}, \ldots k_{\mathrm{d}}\right)}\left(\mathcal{A}_{1}, \mathcal{A}_{2}, \ldots, \mathcal{A}_{n}\right)$

$=N C P M S M^{\left(k_{1}, k_{2}, \ldots k_{d}\right)}\left(\mathcal{A}_{1}^{\prime}, \mathcal{A}_{2}^{\prime}, \ldots, \mathcal{A}_{n}^{\prime}\right)$.

Theorem 3. (Idempotency) Assume that $\mathcal{A}_{1}, \mathcal{A}_{2}, \ldots, \mathcal{A}_{n}(i=1,2, \ldots, n)$ be a set of NCNs. If $\mathcal{A}_{i}=\mathcal{A}$ for all i, then $\operatorname{NCPMSM}^{(\mathrm{k})}\left(\mathcal{A}, \mathcal{A}_{2}, \ldots, \mathcal{A}_{n}\right)=\mathcal{A}$.

Proof. Due to $\mathcal{A}_{i}=\mathcal{A}$ for all i, according to Definition 17 we can get

$$
\begin{aligned}
& \operatorname{NCPMSM}^{\left(k_{1}, k_{2}, \ldots k_{d}\right)}\left(\mathcal{A}_{1}, \mathcal{A}_{2}, \ldots, \mathcal{A}_{n}\right)
\end{aligned}
$$

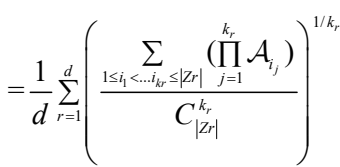

$$
\begin{aligned}
& =\frac{1}{d} \sum_{r=1}^{d}\left(\frac{\sum_{1 \leqslant i_{i}<\ldots i_{r} \leq\left|z r^{\prime}\right|}\left(\prod_{j=1}^{k_{r}} \mathcal{A}\right)}{C_{\left|z r_{r}\right|}^{k_{r}}}\right)^{1 / k_{r}} \\
& =\frac{1}{d} \sum_{r=1}^{d}\left(\frac{\sum_{1 \leq i_{1}<\ldots i_{s} \leq\left|r_{r}\right|} \mathcal{A}^{k_{r}}}{C_{\left|z r_{r}\right|}^{k_{r} \mid}}\right)^{1 / k_{r}} \\
& =\frac{1}{d} \sum_{r=1}^{d}\left(\frac{C_{|z i|}^{k_{r}} \mathcal{A}^{k_{r}}}{C_{\mid z r_{r}}^{k_{r}}}\right)^{1 / k_{r}} \\
& =\frac{1}{d} \sum_{r=1}^{d} \mathcal{A} \\
& =\mathcal{A}
\end{aligned}
$$

So we can get $\operatorname{NCPMSM}^{(\mathrm{k})}\left(\mathcal{A}, \mathcal{A}_{2}, \ldots, \mathcal{A}_{n}\right)=\mathcal{A}$.

Theorem 4. (Monotonicity) Assume $\mathcal{A}_{\mathrm{i}}=\left\{<\left[\mathrm{A}_{\mathrm{Ti}}^{\mathrm{L}}, \mathrm{A}\right.\right.$ $\left.\left.{ }_{\mathrm{Ti}}^{\mathrm{U}}\right],\left[\mathrm{A}_{\mathrm{Ii}}^{\mathrm{L}}, \mathrm{A}_{\mathrm{Ii}}^{\mathrm{U}}\right],\left[\mathrm{A}_{\mathrm{Fi}}^{\mathrm{L}}, \mathrm{A}_{\mathrm{Fi}}^{\mathrm{U}}\right]>,<\lambda_{\mathrm{Ti}}, \lambda_{\mathrm{Ii}}, \lambda_{\mathrm{Fi}}>\right\}$ and $\mathcal{B}_{\mathrm{i}}=$
$\left\{<\left[\mathrm{B}_{\mathrm{Ti}}^{\mathrm{L}}, \mathrm{B}_{\mathrm{Ti}}^{\mathrm{U}}\right],\left[\mathrm{B}_{\mathrm{Ii}}^{\mathrm{L}}, \mathrm{B}_{\mathrm{Ii}}^{\mathrm{U}}\right],\left[\mathrm{B}_{\mathrm{Fi}}^{\mathrm{L}}, \mathrm{B}_{\mathrm{Fi}}^{\mathrm{U}}\right]>,<\lambda_{\mathrm{Ti}}{ }^{\prime}, \lambda_{\mathrm{Ii}}{ }^{\prime}, \lambda\right.$ $\left.\mathrm{Fi}^{\prime}>\right\}(\mathrm{i}=1,2, \ldots \mathrm{n})$ as two collections of NCNs. If $\mathcal{A}_{\mathrm{i}} \leq \mathcal{B}_{\mathrm{i}}$, i.e. $\mathrm{A}_{\mathrm{Ti}}^{\mathrm{L}} \leq \mathrm{B}_{\mathrm{Ti}}^{\mathrm{L}}, \mathrm{A}_{\mathrm{Ti}}^{\mathrm{U}} \leq \mathrm{B}_{\mathrm{Ti}}^{\mathrm{U}}, \mathrm{A}_{\mathrm{li}}^{\mathrm{L}} \geq \mathrm{B}_{\mathrm{li}}^{\mathrm{L}}, \mathrm{A}$ $\geq \mathrm{B}_{\mathrm{Ii}}^{\mathrm{U}}, \mathrm{A}_{\mathrm{Fi}}^{\mathrm{L}} \geq \mathrm{B}_{\mathrm{Fi}}^{\mathrm{L}}, \mathrm{A}_{\mathrm{Fi}}^{\mathrm{U}} \geq \mathrm{B}_{\mathrm{Fi}}^{\mathrm{U}}, \lambda_{\mathrm{Ti}} \leq \lambda_{\mathrm{Ti}}{ }^{\prime}, \lambda_{\mathrm{Ii}} \geq \lambda_{\mathrm{Ii}}{ }^{\prime}$, $\lambda \quad \mathrm{Fi} \geq \lambda \quad \mathrm{Fi}^{\prime}, \quad$ then $\operatorname{NCPMSM}^{\left(k_{1}, k_{2}, \ldots k_{\mathrm{d}}\right)}\left(\mathcal{A}_{1}, \mathcal{A}_{2}, \ldots, \mathcal{A}_{n}\right) \leq \operatorname{NCPMSM}^{\left(k_{1}, k_{2}, \ldots k_{\mathrm{d}}\right)}$ $\left(\mathcal{B}_{1}, \mathcal{B}_{2}, \ldots, \mathcal{B}_{n}\right)$.

Proof. From Definition 15 we know that $d \geq 1$, $\mathrm{k}_{\mathrm{r}} \geq 1$ and $\mathrm{C}_{|\mathrm{zr}|}^{\mathrm{kr}} \geq 1$.

(1) Due to $A_{T_{i}}^{L} \leq B_{T_{i}}^{L}$, we can get $1-\prod_{j=1}^{k_{r}} A_{T_{i_{j}}}^{L} \geq$

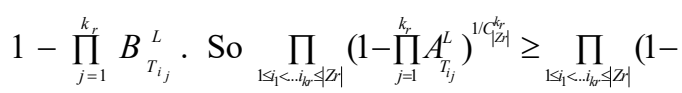

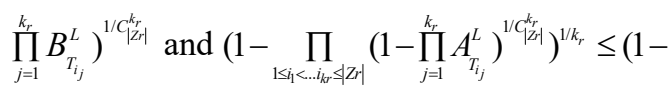
$\left.\prod_{1 \leq i_{i} \leq \ldots i_{r} \leq\left\langle z r_{1}\right|}\left(1-\prod_{j=1}^{k_{r}} B_{T_{i_{j}}}^{L}\right)^{1 / C_{\not>-}^{k_{r}}}\right)^{1 / k_{r}}$. Finally we get

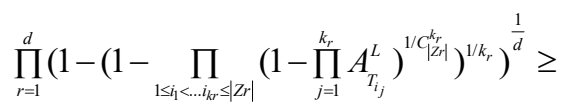

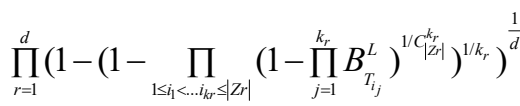
and

$$
\begin{aligned}
& 1-\prod_{r=1}^{d}\left(1-\left(1-\prod_{1 \leq i_{1}<\ldots i_{r r} \leq\left|Z r_{r}\right|}\left(1-\prod_{j=1}^{k_{r}} A_{T_{i_{j}}^{L}}^{L}\right)^{1 / k_{|2 r|}^{k_{r} \mid}}\right)^{1 / k_{r}}\right)^{\frac{1}{d}} \leq \\
& 1-\prod_{r=1}^{d}\left(1-\left(1-\prod_{1 \leq i_{1}<\ldots, i_{k r} \leq|Z r|}\left(1-\prod_{j=1}^{k_{r}} B_{T_{i_{j}}}^{L}\right)^{1 / C_{|r r|}^{k_{r}}}\right)^{1 / k_{r}}\right)^{\frac{1}{d}} .
\end{aligned}
$$

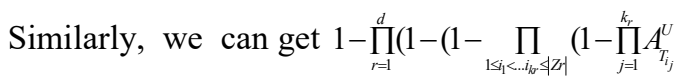

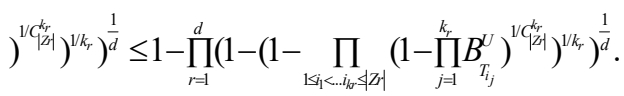

(2) Since $A_{I_{i}}^{L} \geq B_{I_{i}}^{L}$, we can get $1-A_{I_{i_{j}}}^{L} \leq 1-B_{I_{I_{j}}}^{L}$

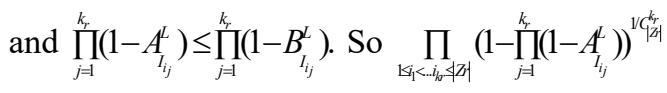

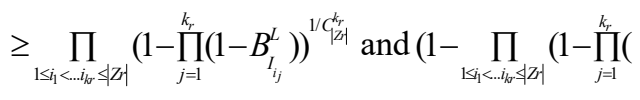

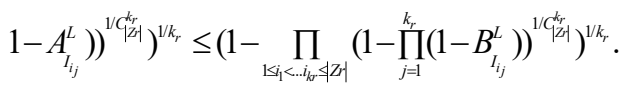

Finally we can get 


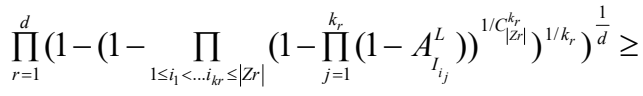

$$
\begin{aligned}
& \prod_{r=1}^{d}\left(1-\left(1-\prod_{1 \leq i_{1}<\ldots, i_{r_{r}} \leq|z r|}\left(1-\prod_{j=1}^{k_{r}}\left(1-B_{I_{i_{j}}}^{L}\right)\right)^{1 /\left.\right|_{\mid Z i} ^{k_{r}}}\right)^{1 / k_{r}}\right)^{\frac{1}{d}} .
\end{aligned}
$$

Similarly,

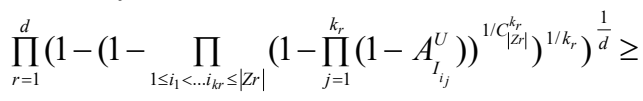

$$
\begin{aligned}
& \prod_{r=1}^{d}\left(1-\left(1-\prod_{1 \leq i_{1}<\ldots, i_{k r} \leq|z r|}\left(1-\prod_{j=1}^{k_{r}}\left(1-B_{I_{i_{j}}}^{U}\right)\right)^{1 /\left.\right|_{\left|k_{i}\right|} ^{k_{r}}}\right)^{1 / k_{r}}\right)^{\frac{1}{d}} .
\end{aligned}
$$

(3) Since $A_{F_{i}}^{L} \geq B_{F_{i}}^{L}, A_{F_{i}}^{U} \geq B_{F_{i}}^{U}$, according to (2), we get

$$
\begin{aligned}
& \prod_{r=1}^{d}\left(1-\left(1-\prod_{1 \leq i_{i}<\ldots, i_{r r} \leq\{z r \mid}\left(1-\prod_{j=1}^{k_{r}}\left(1-A_{F_{i_{j}}}^{L}\right)\right)^{1 /\left.\right|_{\not<-1} ^{k_{r}}}\right)^{1 / k_{r}}\right)^{\frac{1}{d}} \geq \\
& \prod_{r=1}^{d}\left(1-\left(1-\prod_{1 \leq i_{1}<\ldots, i_{r_{r}} \leq|z r|}\left(1-\prod_{j=1}^{k_{r}}\left(1-B_{F_{i_{j}}}^{L}\right)\right)^{1 / c_{|z| r \mid}^{k_{r}}}\right)^{1 / k_{r}}\right)^{\frac{1}{d}}
\end{aligned}
$$

and

$$
\begin{aligned}
& \prod_{r=1}^{d}\left(1-\left(1-\prod_{1 \leq i_{i}<\ldots, i_{r r} \leq\langle 2 r|}\left(1-\prod_{j=1}^{k_{r}}\left(1-A_{F_{i_{j}}}^{U}\right)\right)^{1 /\left.\right|_{|\not i|} ^{k_{r}}}\right)^{1 / k_{r}}\right)^{\frac{1}{d}} \geq \\
& \prod_{r=1}^{d}\left(1-\left(1-\prod_{1 \leq i_{1}<\ldots i_{r} \leq|z r|}\left(1-\prod_{j=1}^{k_{r}}\left(1-B_{F_{i_{j}}}^{U}\right)\right)^{1 /\left.\right|_{|\not i|} ^{k_{r}}}\right)^{1 / k_{r}}\right)^{\frac{1}{d}} .
\end{aligned}
$$

(4) Since $\lambda_{T_{i}} \leq \lambda_{T_{i}}^{\prime}, \lambda_{I_{i}} \geq \lambda_{I_{i}^{\prime}}^{\prime}, \lambda_{F_{i}} \geq \lambda_{F_{i}}^{\prime}$, according to (1), (2) and (3), we can get

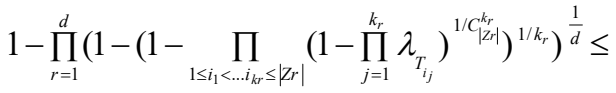

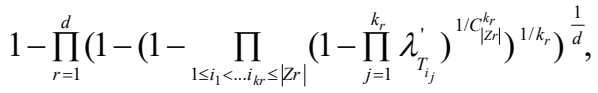

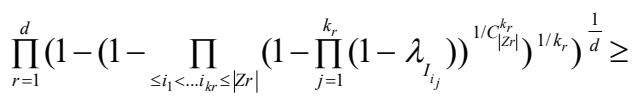

$$
\begin{aligned}
& \prod_{r=1}^{d}\left(1-\left(1-\prod_{\leq i_{1}<\ldots, i_{k r} \leq\left|Z r_{r}\right|}\left(1-\prod_{j=1}^{k_{r}}\left(1-\lambda_{I_{i_{j}}^{\prime}}\right)\right)^{1 / c_{\mid z-1}^{k_{r}}}\right)^{1 / k_{r}}\right)^{\frac{1}{d}},
\end{aligned}
$$

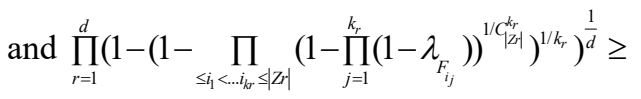

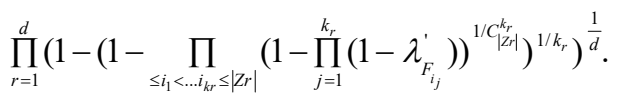

In summary, we prove $N C P M S M^{\left(k_{1}, k_{2}, \ldots, k_{d}\right)}\left(\mathcal{A}_{1}, \mathcal{A}_{2}, \ldots, \mathcal{A}_{n}\right)$ $\leq \operatorname{NCPMSM}^{\left(k_{1}, k_{2}, \ldots k_{d}\right)}\left(\mathcal{B}_{1}, \mathcal{B}_{2}, \ldots, \mathcal{B}_{n}\right)$.
Theorem 5. (Boundedness) Assume that $\mathcal{A}_{1}, \mathcal{A}_{2}, \ldots, \mathcal{A}_{n}(\mathrm{i}=1,2, \ldots \mathrm{n})$ be a set of NCNs and $\mathcal{A}_{\mathrm{i}}=\left\{<\left[\mathrm{A}_{\mathrm{Ti}}^{\mathrm{L}}, \mathrm{A}_{\mathrm{Ti}}^{\mathrm{U}}\right],\left[\mathrm{A}_{\mathrm{Ii}}^{\mathrm{L}}, \mathrm{A}_{\mathrm{Ii}}^{\mathrm{U}}\right],\left[\mathrm{A}_{\mathrm{Fi}}^{\mathrm{L}}, \mathrm{A}_{\mathrm{Fi}}^{\mathrm{U}}\right]>,<\lambda_{\mathrm{Ti}}, \lambda_{\mathrm{Ii}}\right.$, $\left.\lambda_{\mathrm{Fi}}>\right\}$. If

$\mathcal{A}^{-}=\left\{\left\langle\left[\min A_{T_{i}}^{L}, \min A_{T_{i}}^{U}\right],\left[\max A_{I_{i}}^{L}, \max A_{I_{i}}^{U}\right],\left[\max A_{F_{i}}^{L}, \max A_{F_{i}}^{U}\right]\right\rangle\right.$, $\left.\left\langle\min \lambda_{T_{i}}, \max \lambda_{I_{i}}, \max \lambda_{F_{i}}\right\rangle\right\}$, $\mathcal{A}^{+}=\left\{\left[\left[\max A_{T_{i}}^{L}, \max A_{T_{i}}^{U}\right],\left[\min A_{I_{i}}^{L}, \min A_{I_{i}}^{U}\right],\left[\min A_{F_{i}}^{L}, \min A_{F_{i}}^{U}\right]\right\rangle\right.$, $\left.\left\langle\max \lambda_{T_{i}}, \min \lambda_{I_{i}}, \min \lambda_{F_{i}}\right\rangle\right\}$.

Then $\mathcal{A}^{-} \leq N C P M S M^{\left(k_{1}, k_{2}, \ldots k_{d}\right)}\left(\mathcal{A}_{1}, \mathcal{A}_{2}, \ldots, \mathcal{A}_{n}\right) \leq \mathcal{A}^{+}$.

Proof. According to the idempotency and monotonicity of the NCPMSM operator, we can derive the following inequalities:

$N C P M S M^{\left(k_{1}, k_{2}, k_{1}\right)}(\mathcal{A}, \mathcal{A}, \ldots, \mathcal{A}) \geq N C P M S M^{\left.k_{1}, k_{2}, \ldots k_{1}\right)}(\mathcal{A}, \mathcal{A}, \ldots$, $\mathcal{A})=\mathcal{A}$, $N C P M S M^{\left(k_{1}, k_{2}, \ldots k_{1}\right)}\left(\mathcal{A}, \mathcal{A}_{2}, \ldots, \mathcal{A}_{n}\right) \leq N C P M S M^{\left(k_{1}, k_{2}, \ldots k_{1}\right)}\left(\mathcal{A}^{+}, \mathcal{A}^{+}, \ldots\right.$, $\left.\mathcal{A}^{+}\right)=\mathcal{A}^{+}$.

So, $\mathcal{A}^{-} \leq N C P M S M^{\left(k_{1}, k_{2}, \ldots k_{d}\right)}\left(\mathcal{A}_{1}, \mathcal{A}_{2}, \ldots, \mathcal{A}_{n}\right) \leq \mathcal{A}^{+}$.

Definition 18. Let $\mathcal{A}_{1}, \mathcal{A}_{2}, \ldots, \mathcal{A}_{n}$ be a set of NCNs where $\mathcal{A}_{\mathrm{i}}=\left\{<\left[\mathrm{A}_{\mathrm{Ti}}^{\mathrm{L}}, \mathrm{A}_{\mathrm{Ti}}^{\mathrm{U}}\right],\left[\mathrm{A}_{\mathrm{Ii}}^{\mathrm{L}}, \mathrm{A}_{\mathrm{Ii}}^{\mathrm{U}}\right],\left[\mathrm{A}_{\mathrm{Fi}}^{\mathrm{L}}, \mathrm{A}\right.\right.$ $\left.{ }_{\mathrm{Fi}}^{\mathrm{U}}\right]>, \quad\left\langle\lambda_{\mathrm{Ti}}, \lambda_{\mathrm{Ii}}, \lambda_{\mathrm{Fi}}>\right\}$. And let the weight of the input argument $\mathcal{A}_{i}(\mathrm{i}=1,2, \ldots, \mathrm{n})$ be $\mathrm{w}_{\mathrm{i}}$, where $\mathrm{w}_{\mathrm{i}} \in[0,1]$ and $\sum_{i}^{\mathrm{n}} \mathrm{w}_{\mathrm{i}}=1$. Then the neutrosophic cubic weighted partitioned Maclaurin symmetric mean (NCWPMSM) operator is defined as follows:

$$
\begin{aligned}
& N C W P M S M^{\left(k_{1}, k_{2}, \ldots k_{d}\right)}\left(\mathcal{A}_{1}, \mathcal{A}_{2}, \ldots, \mathcal{A}_{n}\right) \\
& =\frac{1}{d} \sum_{r=1}^{d}\left(\frac{\sum_{1 i_{i}<\ldots i_{r} \leq|z r r|}\left(\prod_{j=1}^{k_{r}} w_{i_{j}} \mathcal{A}_{i_{j}}\right)}{C_{\left|z r_{r}\right|}^{k_{r}}}\right)^{1 / k_{r}},
\end{aligned}
$$

where $\mathrm{k}_{\mathrm{r}}$ is the parameter in the partition $\mathrm{Z}_{\mathrm{r}}, \mathrm{k}=1$, $2, \ldots,\left|Z_{r}\right| .\left|Z_{r}\right|$ is the number of input arguments in the partition $Z_{r}$ and $\sum_{r=1}^{d}|Z r|=N .\left(i_{1}, i_{2}, \ldots, i_{k}\right)$ traverses the overall $\mathrm{k}_{\mathrm{r}}$ - tuple different combinations of $\left(1,2, \ldots,\left|Z_{r}\right|\right) ; C_{|Z r|}^{k r}$ denotes the binomial coefficient and it satisfies: 


$$
C_{|Z r|}^{k r}=\frac{\left|Z_{r}\right| !}{k_{r} !\left(\left|Z_{r}\right|-k r\right) !} .
$$

Theorem 6. Let $\mathcal{A}_{1}, \mathcal{A}_{2}, \ldots \mathcal{A}_{n}$ be a set of NCNs and the weight of the input argument $\mathcal{A}_{i}(\mathrm{i}=1,2, \ldots, \mathrm{n})$ be $\mathrm{w}_{\mathrm{i}}$, where $\mathrm{w}_{\mathrm{i}} \in[0,1]$ and $\sum_{i}{ }^{\mathrm{n}} \mathrm{w}_{\mathrm{i}}=1$. Then the integrated result of the above NCWPMSM operator is also a NCN, displayed as below:

$$
\begin{aligned}
& N_{C W P M S M}{ }^{\left(k_{1}, k_{2}, \ldots k_{d}\right)}\left(\mathcal{A}_{1}, \mathcal{A}_{2}, \ldots, \mathcal{A}_{n}\right) \\
& =\frac{1}{d} \sum_{r=1}^{d}\left(\frac{\sum_{1 \leq i_{1}<\ldots i_{r} \leq \leq|z|}\left(\prod_{j=1}^{k_{r}} w_{i_{j}} \mathcal{A}_{i_{j}}\right)}{C_{|Z r|}^{k_{r}}}\right)^{1 / k_{r}}
\end{aligned}
$$

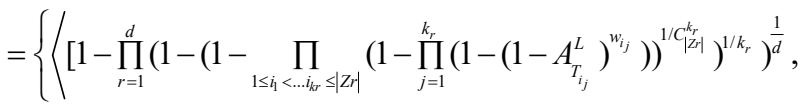

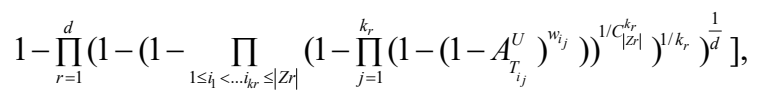

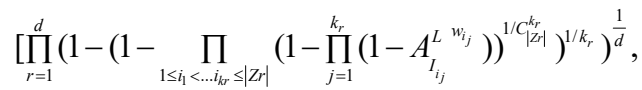

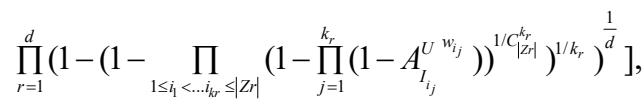

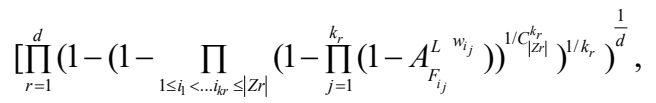

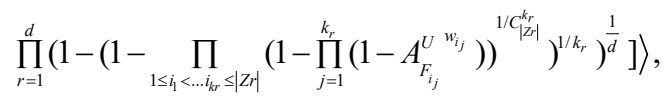

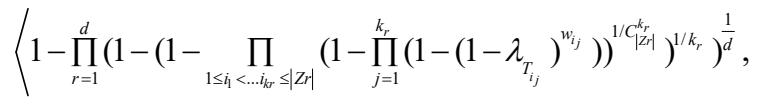

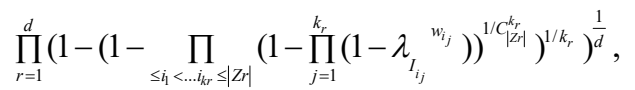

$$
\begin{aligned}
& \left.\left.\prod_{r=1}^{d}\left(1-\left(1-\prod_{\leq i_{i}<\ldots i_{r} \leq|\leq r|}\left(1-\prod_{j=1}^{k_{r}}\left(1-\lambda_{F_{i_{j}}}{ }_{F_{i j}}^{w_{i j}}\right)\right)^{1 / c_{\mid r_{i n}}^{k_{r}}}\right)^{1 / k_{r}}\right)^{\frac{1}{d}}\right\rangle\right\} .
\end{aligned}
$$

Similar to the proof of theorem 1, we omit this process of proof here.

Same as the NCPMSM operator, the NCWPMSM operator also has some properties of commutativity, idempotency, monotonicity and boundedness. The processes of proof are similar to Theorem 2 Theorem 5, so here we omit these proofs.

We can use the score function of IFV in definition 3 to acquire the score of NCN. In order to meet the expected properties of the score function, we have improved it.

Definition 19. For a neutrosophic cubic number $\mathcal{A}=\left\{<\left[\mathrm{A}_{\mathrm{T}}^{\mathrm{L}}, \mathrm{A}_{\mathrm{T}}^{\mathrm{U}}\right],\left[\mathrm{A}_{\mathrm{I}}^{\mathrm{L}}, \mathrm{A}_{\mathrm{I}}^{\mathrm{U}}\right],\left[\mathrm{A}_{\mathrm{F}}^{\mathrm{L}}, \mathrm{A}_{\mathrm{F}}^{\mathrm{U}}\right]>,<\lambda_{\mathrm{T}}, \lambda_{\mathrm{I}}, \lambda\right.$ $\left.\mathrm{F}^{>}\right\}$, the proposed novel score function of $\mathrm{NCN}$ of $\mathcal{A}$ is as follows:

$$
\begin{aligned}
S(\mathcal{A})= & \frac{1}{3}\left(A_{T}^{L}+A_{T}^{U}+\lambda_{T}-A_{F}^{L}-A_{F}^{U}-\lambda_{F}-A_{I}^{L} \times \frac{A_{I}^{L}-\log _{2}\left(1+A_{I}^{L}\right)}{100}\right. \\
& \left.-A_{I}^{U} \times \frac{A_{I}^{U}-\log _{2}\left(1+A_{I}^{U}\right)}{100}-\lambda_{I} \times \frac{\lambda_{I}-\log _{2}\left(1+\lambda_{I}\right)}{100}\right) .
\end{aligned}
$$

This new function can make up for some shortcomings of the existing score function in [29]. For example, $\mathcal{A}=\{<[0.8,0.9],[0.5,0.6]$, $[0.4,0.6],<0.7,0.5,0.6>\}, \mathcal{B}=\{<[0.7,0.8]$, $[0.4,0.7],[0.6,0.7],<0.6,0.2,0.3>\}$, according to the existing score function (Ye 2018) we can get $\mathrm{S}(\mathrm{A})=\mathrm{S}(\mathrm{B})=0.58$. Apparently, the original score function can't accurately distinguish the size of two numbers. Based on the novel score function, we get $\mathrm{S}(\mathrm{A})=0.2671$, $\mathrm{S}(\mathrm{B})=0.1670$, i.e. $\mathrm{A}>\mathrm{B}$.

Theorem 7. The proposed novel score function of NCN satisfies: $S(\mathcal{A}) \in[-1,1]$.

Proof. Let $\mathcal{A}=\left\{<\left[\mathrm{A}_{\mathrm{T}}^{\mathrm{L}}, \mathrm{A}_{\mathrm{T}}^{\mathrm{U}}\right],\left[\mathrm{A}_{\mathrm{I}}^{\mathrm{L}}, \mathrm{A}_{\mathrm{I}}^{\mathrm{U}}\right],\left[\mathrm{A}_{\mathrm{F}}^{\mathrm{L}}, \mathrm{A}_{\mathrm{F}}^{\mathrm{U}}\right]>\right.$, $\left.<\lambda_{\mathrm{T}}, \lambda_{\mathrm{I}}, \lambda_{\mathrm{F}}>\right\}$ and $\mathcal{B}=\left\{<\left[\mathrm{B}_{\mathrm{T}}^{\mathrm{L}}, \mathrm{B}_{\mathrm{T}}^{\mathrm{U}}\right],\left[\mathrm{B}_{\mathrm{I}}^{\mathrm{L}}, \mathrm{B}_{\mathrm{I}}^{\mathrm{U}}\right],\left[\mathrm{B}_{\mathrm{F}}^{\mathrm{L}}\right.\right.$, $\left.\left.\mathrm{B}_{\mathrm{F}}^{\mathrm{U}}\right]>,<\lambda_{\mathrm{T}}{ }^{\prime}, \lambda_{\mathrm{I}}{ }^{\prime}, \lambda_{\mathrm{F}}{ }^{\prime}>\right\}$ be any two NCNs. Assume that $\mathcal{A}>\mathcal{B}$, i.e. $\mathrm{A}_{\mathrm{T}}^{\mathrm{L}}>\mathrm{B}_{\mathrm{T}}^{\mathrm{L}}, \mathrm{A}_{\mathrm{T}}^{\mathrm{U}}>\mathrm{B}_{\mathrm{T}}^{\mathrm{U}}, \mathrm{A}_{\mathrm{I}}^{\mathrm{L}}$ $<\mathrm{B}_{\mathrm{I}}^{\mathrm{L}}, \mathrm{A}_{\mathrm{I}}^{\mathrm{U}}<\mathrm{B}_{\mathrm{I}}^{\mathrm{U}}, \mathrm{A}_{\mathrm{F}}^{\mathrm{L}}<\mathrm{B}_{\mathrm{F}}^{\mathrm{L}}, \mathrm{A}_{\mathrm{F}}^{\mathrm{U}}<\mathrm{B}_{\mathrm{F}}^{\mathrm{U}}, \lambda_{\mathrm{T}}>\lambda_{\mathrm{T}}{ }^{\prime}, \lambda_{\mathrm{I}}$ $<\lambda_{\mathrm{I}}{ }^{\prime}, \lambda_{\mathrm{Fi}}<\lambda_{\mathrm{F}}{ }^{\prime}$. Based on definition 19, we can get

$$
\begin{aligned}
S(\mathcal{A})-S(B)= & \frac{1}{3}\left(\left(A_{T}^{L}-B_{T}^{L}\right)+\left(A_{T}^{U}-B_{T}^{U}\right)+\left(\lambda_{T}-\lambda_{T}^{\prime}\right)\right. \\
& -\left(A_{F}^{L}-B_{F}^{L}\right)-\left(A_{F}^{U}-B_{F}^{U}\right)-\left(\lambda_{F}-\lambda_{F}^{\prime}\right) \\
& -\left(A_{I}^{L} \times \frac{A_{I}^{L}-\log _{2}\left(1+A_{I}^{L}\right)}{100}-B_{I}^{L} \times \frac{B_{I}^{L}-\log _{2}\left(1+B_{I}^{L}\right)}{100}\right) \\
& -\left(A_{I}^{U} \times \frac{A_{I}^{U}-\log _{2}\left(1+A_{I}^{U}\right)}{100}-B_{I}^{U} \times \frac{B_{I}^{U}-\log _{2}\left(1+B_{I}^{U}\right)}{100}\right) \\
& \left.-\left(\lambda_{I} \times \frac{\lambda_{I}-\log _{2}\left(1+\lambda_{I}\right)}{100}-\lambda_{I}^{\prime} \times \frac{\lambda_{I}^{\prime}-\log _{2}\left(1+\lambda_{I}^{\prime}\right)}{100}\right)\right)
\end{aligned}
$$

Apparently, $\mathrm{A}_{\mathrm{T}}^{\mathrm{L}}-\mathrm{B}_{\mathrm{T}}^{\mathrm{L}}>0, \mathrm{~A}_{\mathrm{T}}^{\mathrm{U}}-\mathrm{B}_{\mathrm{T}}^{\mathrm{U}}>0$, - $\left(\mathrm{A}_{\mathrm{F}}^{\mathrm{L}}-\right.$ $\left.\mathrm{B}_{\mathrm{F}}^{\mathrm{L}}\right)>0,-\left(\mathrm{A}_{\mathrm{F}}^{\mathrm{U}}-\mathrm{B}_{\mathrm{F}}^{\mathrm{U}}\right)>0, \lambda_{\mathrm{F}}-\lambda_{\mathrm{F}}{ }^{\prime}>0$. Now we construct a function $\mathrm{y}$ : 


$$
\begin{aligned}
y & =x \times\left(x-\log _{2}(1+x)\right) \\
& =x^{2}-x \log _{2}(1+x), \mathrm{x} \in[0,1] \\
y^{\prime} & =2 x-\log _{2}(1+x)-\frac{x}{(1+x) \ln 2} \\
& =2 x-\log _{2}(1+x)+\frac{1}{(1+x) \ln 2}-\frac{1}{\ln 2}, \mathrm{x} \in[0,1] \\
y^{\prime \prime} & =2-\frac{1}{(1+x) \ln 2}-\frac{1}{(1+x)^{2} \ln 2}, \mathrm{x} \in[0,1]
\end{aligned}
$$

It is easy to see that $\mathrm{y}^{\prime \prime}$ is a decreasing function with respect to $\mathrm{x}$. So

$$
y_{\text {min }}^{\prime \prime}=y^{\prime \prime}(x=1)=2-\frac{1}{(1+1) \ln 2}-\frac{1}{(1+1)^{2} \ln 2}=2-\frac{3}{4 \ln 2}>0
$$

So $\mathrm{y}^{\prime}$ is an increasing function with respect to $\mathrm{x}$ and

$$
y_{\min }^{\prime}=y^{\prime}(x=0)=2 \times 0-\log _{2}(1+0)+\frac{1}{(1+0) \ln 2}-\frac{1}{\ln 2}=0 \text {. }
$$

Apprantly, $\mathrm{y}$ is an increasing function with respect to $\mathrm{x}$. So

$$
\begin{gathered}
-\left(A_{F}^{L} \times \frac{A_{F}^{L}-\log _{2}\left(1+A_{F}^{L}\right)}{100}-B_{F}^{L} \times \frac{B_{F}^{L}-\log _{2}\left(1+B_{F}^{L}\right)}{100}\right)>0, \\
-\left(A_{F}^{U} \times \frac{A_{F}^{U}-\log _{2}\left(1+A_{F}^{U}\right)}{100}-B_{F}^{U} \times \frac{B_{F}^{U}-\log _{2}\left(1+B_{F}^{U}\right)}{100}\right)>0, \\
-\left(\lambda_{I} \times \frac{\lambda_{I}-\log _{2}\left(1+\lambda_{I}\right)}{100}-\lambda_{I}^{\prime} \times \frac{\lambda_{I}^{\prime}-\log _{2}\left(1+\lambda_{I}^{\prime}\right)}{100}\right)>0 .
\end{gathered}
$$

so we know $S(\mathcal{A})-S(B)>0$, i.e. $S(\mathcal{A})>S(B)$.

Let $\mathcal{A}_{1}=\{<[1,1],[0,0],[0,0]>,<1,0,0>\}$ and $\mathcal{A}_{2}=\{<[0,0],[1,1],[1,1]>,<0,1,1>\}, \mathcal{A}_{1}$ and $\mathcal{A}_{2}$ are the maximum and minimum values of the $\mathrm{NCN}$, respectively. So

$$
\begin{aligned}
S(\max ) & =S_{C K}\left(\mathcal{A}_{1}\right)=\frac{1}{3}\left(1+1+1-0-0-0-0 \times \frac{0-\log _{2}(1+0)}{100}\right. \\
& \left.-0 \times \frac{0-\log _{2}(1+0)}{100}-0 \times \frac{0-\log _{2}(1+0)}{100}\right) \\
& =1 \\
S(\min ) & =S_{C K}\left(\mathcal{A}_{2}\right)=\frac{1}{3}\left(0+0+0-1-1-1-1 \times \frac{1-\log _{2}(1+1)}{100}\right. \\
& \left.-1 \times \frac{1-\log _{2}(1+1)}{100}-1 \times \frac{1-\log _{2}(1+1)}{100}\right) \\
& =-1
\end{aligned}
$$

Therefore, we can get $\mathrm{S} \in[-1,1]$

\section{A Multi-attribute group decision-making method based on the NCWPMSM operator and a novel score function}

For neutrosophic cubic multi-attribute group decision-making method, supposing there are $\mathrm{m}$ alternatives $\mathrm{A}=\left\{\mathrm{A}_{1}, \mathrm{~A}_{2}, \ldots, \mathrm{A}_{\mathrm{i}}, \ldots, \mathrm{A}_{\mathrm{m}}\right\}$ and $\mathrm{n}$ attributes $C=\left\{C_{1}, C_{2}, \ldots, C_{j}, \ldots, C_{n}\right\}$. The weights of attributes are $\mathrm{w}=\left(\mathrm{w}_{1}, \mathrm{w}_{2}, \ldots, \mathrm{w}_{\mathrm{j}}, \ldots, \mathrm{w}_{\mathrm{n}}\right)$. There are 1 experts $D^{k}(k=1,2, \ldots 1)$, whose weights are $\gamma=\left(\gamma_{1}, \gamma_{2}, \ldots \gamma_{1}\right)$, are invited to evaluate the alternatives. The concret decision-making process is shown as follows:

Step1. Construct the decision matrix $\mathrm{R}^{\mathrm{k}}$

All experts give their own evaluation values about this problem. The evaluation value of $\mathrm{A}_{\mathrm{i}}$ on attribute $\mathrm{C}_{\mathrm{j}}$ given by expert $\mathrm{k}$ is represented by the decision matrix $R^{k}=\left(r_{i j}^{k}\right)_{m \times n}$, where $r_{i j}$ is a NCN.

Step2. Aggregate information from each expert

Use the NCWAA operator in Definition 11 to gather decision information from all experts:

$$
r_{i j}=\operatorname{NCWAA}\left(r_{i j}^{1}, r_{i j}^{2}, \ldots, r_{i j}^{l}\right) \text {. }
$$

Step3. Obtain the comprehensive evaluation value

Firstly, we need to partition the attributes. Then we need to use the NCWPMSM operator in definition 18 to integrate the evaluation information of different attribute values of each alternative to obtain the comprehensive evaluation value.

Step4. Calculate the score function for the alternatives

We can use the score function in definition 19 to acquire the score values of alternatives.

Step5. Rank all the alternatives.

This step we need to rank all the alternatives according to respective score value in decending order. The larger the score value, the better the alternative. 


\section{An application example}

Supplier selection is an important part of the industry and production strategy for industrial organizations. Choosing the best supplier will improve the overall efficiency and cost effectiveness of the whole supply chain. Consider a decision problem in an automobile manufacturing enterprise, which aims to find a suitable supplier for the purchase of components. After preliminary screening, four potential automobile equipment suppliers $\left(A_{1}, A_{2}, A_{3}, A_{4}\right)$ have been identified for further evaluation. Five attributes to be considered in the evaluation process are: $\mathrm{C}_{1}$ : Environment management; $\mathrm{C}_{2}$ : Quality management; $\mathrm{C}_{3}$ : Product performance; $\mathrm{C}_{4}$ : Green image; $\mathrm{C}_{5}$ : Green product innovation. The weights of attributes are $\mathrm{w}=(0.24,0.2,0.2,0.17,0.19)$. Three experts assess this problem and the relative importance vector is $\mathrm{W}=(0.4,0.3,0.3)^{\mathrm{T}}$.

Table 1

Decision matrix $\mathrm{R}^{1}$ of the decision-maker $\mathrm{D}^{1}$

\begin{tabular}{|c|c|c|}
\hline & $\mathrm{C}_{1}$ & $\mathrm{C}_{2}$ \\
\hline $\mathrm{A}_{1}$ & $\begin{array}{c}\{<[0.2,0.3],[0.2,0.3] \\
[0.4,0.5]>,<0.3,0.3,0.5>\}\end{array}$ & $\begin{array}{c}\{<[0.3,0.4],[0.2,0.3] \\
[0.1,0.2]>,<0.4,0.2,0.1>\}\end{array}$ \\
\hline $\mathrm{A}_{2}$ & $\begin{array}{c}\{<[0.3,0.4],[0.1,0.2], \\
[0.2,0.3]>,<0.3,0.2,0.3>\}\end{array}$ & $\begin{array}{c}\{<[0.2,0.3],[0.3,0.4], \\
[0.4,0.5]>,<0.2,0.4,0.5>\}\end{array}$ \\
\hline $\mathrm{A}_{3}$ & $\begin{array}{c}\{<[0.3,0.4],[0.1,0.2], \\
[0.1,0.2]>,<0.4,0.1,0.1>\}\end{array}$ & $\begin{array}{c}\{<[0.5,0.6],[0.2,0.3], \\
[0.2,0.3]>,<0.6,0.2,0.3>\}\end{array}$ \\
\hline \multirow[t]{2}{*}{$\mathrm{A}_{4}$} & $\begin{array}{c}\{<[0.4,0.5],[0.1,0.2], \\
[0.2,0.3]>,<0.5,0.1,0.2>\}\end{array}$ & $\begin{array}{c}\{<[0.6,0.7],[0.2,0.3], \\
[0.1,0.2]>,<0.7,0.2,0.1>\}\end{array}$ \\
\hline & $\mathrm{C}_{4}$ & $\mathrm{C}_{5}$ \\
\hline $\mathrm{A}_{1}$ & $\begin{array}{c}\{<[0.4,0.5],[0.1,0.2], \\
[0.3,0.4]>,<0.4,0.2,0.3>\}\end{array}$ & $\begin{array}{c}\{<[0.7,0.9],[0.2,0.4], \\
[0.3,0.4]>,<0.8,0.5,0.2>\}\end{array}$ \\
\hline $\mathrm{A}_{2}$ & $\begin{array}{c}\{<[0.5,0.6],[0.1,0.2], \\
[0.1,0.2]>,<0.6,0.1,0.1>\}\end{array}$ & $\begin{array}{c}\{<[0.5,0.6],[0.3,0.4], \\
[0.3,0.5]>,<0.7,0.3,0.6>\}\end{array}$ \\
\hline $\mathrm{A}_{3}$ & $\begin{array}{c}\{<[0.5,0.6],[0.2,0.3], \\
[0.1,0.2]>,<0.5,0.4,0.2>\}\end{array}$ & $\begin{array}{c}\{<[0.6,0.8],[0.2,0.4], \\
[0.5,0.6]>,<0.7,0.2,0.4>\}\end{array}$ \\
\hline $\mathrm{A}_{4}$ & $\begin{array}{c}\{<[0.3,0.4],[0.2,0.3], \\
[0.1,0.2]>,<0.5,0.2,0.2>\}\end{array}$ & $\begin{array}{c}\{<[0.7,0.9],[0.2,0.3], \\
[0.3,0.5]>,<0.8,0.5,0.2>\}\end{array}$ \\
\hline
\end{tabular}

Table 2

Decision matrix $\mathrm{R}^{2}$ of the decision-maker $\mathrm{D}^{2}$

\begin{tabular}{cccc}
\hline & $\mathrm{C}_{1}$ & $\mathrm{C}_{2}$ & $\mathrm{C}_{3}$ \\
\hline \multirow{2}{*}{$\mathrm{A}_{1}$} & $\{<[0.3,0.4],[0.2,0.3]$, & $\{<[0.5,0.6],[0.3,0.4]$, & $\{<[0.2,0.3],[0.2,0.3]$, \\
& $[0.5,0.6]>,<0.3,0.3,0.6>\}$ & $[0.2,0.3]>,<0.5,0.4,0.3>\}$ & $[0.4,0.5]>,<0.2,0.2,0.5>\}$ \\
$\mathrm{A}_{2}$ & $\{<[0.5,0.6],[0.2,0.3]$, & $\{<[0.4,0.5],[0.1,0.2]$, & $\{<[0.6,0.7],[0.2,0.3]$, \\
& $[0.1,0.2]>,<0.5,0.3,0.1>\}$ & $[0.3,0.4]>,<0.5,0.2,0.3>\}$ & $[0.1,0.2]>,<0.6,0.3,0.2>\}$ \\
$\mathrm{A}_{3}$ & $\{<[0.4,0.6],[0.1,0.3]$, & $\{<[0.6,0.7],[0.1,0.2]$, & $\{<[0.5,0.6],[0.1,0.2]$, \\
& $[0.2,0.4]>,<0.5,0.2,0.3>\}$ & $[0.2,0.3]>,<0.7,0.1,0.2>\}$ & $[0.1,0.2]>,<0.6,0.1,0.1>\}$
\end{tabular}




\begin{tabular}{|c|c|c|}
\hline $\mathrm{A}_{4}$ & $\begin{array}{c}\{<[0.8,0.9],[0.1,0.1], \\
[0.1,0.2]>,<0.9,0.1,0.1>\}\end{array}$ & $\begin{array}{c}\{<[0.4,0.5],[0.1,0.2], \\
[0.2,0.3]>,<0.5,0.1,0.2>\}\end{array}$ \\
\hline & $\mathrm{C}_{4}$ & $\mathrm{C}_{5}$ \\
\hline $\mathrm{A}_{1}$ & $\begin{array}{c}\{<[0.1,0.6],[0.3,0.4] \\
[0.5,0.8]>,<0.5,0.5,0.7>\}\end{array}$ & $\begin{array}{c}\{<[0.2,0.6],[0.4,0.5] \\
[0.3,0.6]>,<0.6,0.5,0.9>\}\end{array}$ \\
\hline $\mathrm{A}_{2}$ & $\begin{array}{c}\{<[0.2,0.5],[0.4,0.9], \\
[0.5,0.8]>,<0.5,0.2,0.7>\}\end{array}$ & $\begin{array}{c}\{<[0.3,0.4],[0.5,0.7] \\
[0.5,0.8]>,<0.6,0.1,0.7>\}\end{array}$ \\
\hline $\mathrm{A}_{3}$ & $\begin{array}{c}\{<[0.6,0.7],[0.3,0.6] \\
[0.3,0.7]>,<0.7,0.5,0.3>\}\end{array}$ & $\begin{array}{c}\{<[0.6,0.8],[0.4,0.6], \\
[0.5,0.6]>,<0.8,0.4,0.6>\}\end{array}$ \\
\hline $\mathrm{A}_{4}$ & $\begin{array}{c}\{<[0.3,0.7],[0.7,0.8], \\
[0.6,0.7]>,<0.4,0.2,0.8>\}\end{array}$ & $\begin{array}{c}\{<[0.4,0.5],[0.5,0.7], \\
[0.8,0.9]>,<0.4,0.6,0.7>\}\end{array}$ \\
\hline
\end{tabular}

Table 3

Decision matrix $\mathrm{R}^{3}$ of the decision-maker $\mathrm{D}^{3}$

\begin{tabular}{cccc}
\hline & $\mathrm{C}_{1}$ & $\mathrm{C}_{2}$ & $\mathrm{C}_{3}$ \\
\hline \multirow{2}{*}{$\mathrm{A}_{1}$} & $\{<[0.2,0.3],[0.1,0.2]$, & $\{<[0.3,0.4],[0.3,0.4]$, & $\{<[0.5,0.6],[0.2,0.3]$, \\
& $[0.3,0.4]>,<0.2,0.1,0.4>\}$ & $[0.5,0.6]>,<0.3,0.4,0.6>\}$ & $[0.3,0.4]>,<0.6,0.3,0.4>\}$ \\
$\mathrm{A}_{2}$ & $\{<[0.3,0.4],[0.1,0.2]$, & $\{<[0.4,0.5],[0.2,0.3]$, & $\{<[0.6,0.7],[0.1,0.2]$, \\
& $[0.2,0.3]>,<0.4,0.1,0.3>\}$ & $[0.3,0.4]>,<0.5,0.2,0.4>\}$ & $[0.2,0.3]>,<0.6,0.2,0.3>\}$ \\
$\mathrm{A}_{3}$ & $\{<[0.4,0.5],[0.1,0.2]$, & $\{<[0.4,0.5],[0.2,0.3]$, & $\{<[0.7,0.8],[0.1,0.2]$, \\
& $[0.1,0.2]>,<0.5,0.1,0.2>\}$ & $[0.1,0.2]>,<0.5,0.2,0.1>\}$ & $[0.2,0.3]>,<0.8,0.1,0.2>\}$ \\
$\mathrm{A}_{4}$ & $\{<[0.5,0.6],[0.2,0.3]$, & $\{<[0.7,0.8],[0.1,0.2]$, & $\{<[0.8,0.9],[0.1,0.2]$, \\
& $[0.1,0.2]>,<0.6,0.2,0.3>\}$ & $[0.2,0.3]>,<0.7,0.1,0.2>\}$ & $[0.1,0.2]>,<0.8,0.2,0.1>\}$ \\
\hline
\end{tabular}

\begin{tabular}{ccc}
\hline & $\mathrm{C}_{4}$ & $\mathrm{C}_{5}$ \\
\hline $\mathrm{A}_{1}$ & $\{<[0.2,0.3],[0.3,0.4]$, & $\{<[0.2,0.3],[0.3,0.5]$, \\
& $[0.4,0.5]>,<0.3,0.4,0.6>\}$ & $[0.4,0.5]>,<0.6,0.4,0.3>\}$ \\
$\mathrm{A}_{2}$ & $\{<[0.5,0.6],[0.1,0.2]$, & $\{<[0.5,0.6],[0.1,0.2]$, \\
& $[0.2,0.3]>,<0.6,0.3,0.2>\}$ & $[0.2,0.3]>,<0.6,0.3,0.3>\}$ \\
$\mathrm{A}_{3}$ & $\{<[0.3,0.4],[0.1,0.2]$, & $\{<[0.5,0.6],[0.4,0.5]$, \\
& $[0.2,0.3]>,<0.3,0.2,0.4>\}$ & $[0.6,0.8]>,<0.4,0.6,0.7>\}$ \\
$\mathrm{A}_{4}$ & $\{<[0.4,0.5],[0.2,0.3]$, & $\{<[0.7,0.8],[0.8,0.9]$, \\
& $[0.5,0.6]>,<0.2,0.3,0.6>\}$ & $[0.6,0.7]>,<0.3,0.5,0.6>\}$ \\
\hline
\end{tabular}

Table 4

Group decision matrix F

\begin{tabular}{cccc}
\hline & $\mathrm{C}_{1}$ & \multicolumn{1}{c}{$\mathrm{C}_{2}$} & $\mathrm{C}_{3}$ \\
\hline \multirow{2}{*}{$\mathrm{A}_{1}$} & $\{<[0.23,0.33],[0.16,0.27]$, & $\{<[0.37,0.47],[0.26,0.36]$, & $\{<[0.36,0.48],[0.15,0.26]$, \\
& $[0.39,0.49]>,<0.27,0.22,0.49>\}$ & $[0.2,0.31]>,<0.41,0.30,0.24>\}$ & $[0.28,0.38]>,<0.46,0.23,0.32>\}$ \\
$\mathrm{A}_{2}$ & $\{<[0.37,0.47],[0.12,0.23]$, & $\{<[0.33,0.43],[0.19,0.30]$, & $\{<[0.45,0.56],[0.16,0.27]$, \\
& $[0.16,0.27]>,<0.40,0.18,0.22>\}$ & $[0.34,0.44]>,<0.40,0.26,0.40>\}$ & $[0.23,0.35]>,<0.45,0.27,0.35>\}$
\end{tabular}




\begin{tabular}{cccc} 
& $\{<[0.36,0.50],[0.10,0.23]$, & $\{<[0.51,0.61],[0.16,0.27]$, & $\{<[0.57,0.68],[0.10,0.20]$, \\
$\mathrm{A}_{3}$ & $[0.12,0.25]>,<0.46,0.12,0.17>\}$ & $[0.16,0.27]>,<0.61,0.16,0.19>\}$ & $[0.16,0.27]>,<0.68,0.10,0.16>\}$ \\
& $\{<[0.59,0.71],[0.12,0.18]$, & $\{<[0.59,0.69],[0.13,0.24]$, & $\{<[0.71,0.82],[0.16,0.27]$, \\
$\mathrm{A}_{4}$ & $[0.13,0.24]>,<0.71,0.12,0.18>\}$ & $[0.15,0.26]>,<0.65,0.13,0.15>\}$ & $[0.13,0.24]>,<0.77,0.20,0.16>\}$ \\
\hline
\end{tabular}

\begin{tabular}{ccc}
\hline & $\mathrm{C}_{4}$ & $\mathrm{C}_{5}$ \\
\hline \multirow{2}{*}{$\mathrm{A}_{1}$} & $\{<[0.26,0.48],[0.19,0.3]$, & $\{<[0.46,0.73],[0.28,0.46]$, \\
& $[0.38,0.53]>,<0.41,0.32,0.48>\}$ & $[0.33,0.48]>,<0.70,0.47,0.35>\}$ \\
$\mathrm{A}_{2}$ & $\{<[0.42,0.57],[0.15,0.31]$, & $\{<[0.45,0.55],[0.25,0.38]$, \\
& $[0.20,0.34]>,<0.57,0.17,0.22>\}$ & $[0.31,0.49]>,<0.64,0.22,0.51>\}$ \\
$\mathrm{A}_{3}$ & $\{<[0.48,0.59],[0.18,0.33]$, & $\{<[0.57,0.75],[0.30,0.48]$, \\
& $[0.17,0.33]>,<0.53,0.35,0.28>\}$ & $[0.53,0.65]>,<0.67,0.34,0.53>\}$ \\
$\mathrm{A}_{4}$ & $\{<[0.33,0.54],[0.29,0.40]$, & $\{<[0.63,0.80],[0.40,0.54]$, \\
& $[0.28,0.40]>,<0.39,0.23,0.42>\}$ & $[0.50,0.66]>,<0.60,0.53,0.40>\}$ \\
\hline
\end{tabular}

5.1. The effect of the parameters $\mathrm{k}_{1}, \mathrm{k}_{2}$ on the this

After fully considering the relationship between attributes, the five attributes are divided into two parts, i.e. $\left\{\mathrm{C}_{1}, \mathrm{C}_{4}, \mathrm{C}_{5}\right\}$ and $\left\{\mathrm{C}_{2}, \mathrm{C}_{3}\right\}$. That is to say, $\left|Z_{1}\right|=3$ and $\left|Z_{2}\right|=2$.

The concrete decision-making procedure is as follows:

Step1. Construct the decision matrix $\mathrm{R}^{\mathrm{k}}$

The experts assign values to the suppliers through the NCNs to form three decision matrices, as shown in Table $1-3$.

Step2. Aggregate information from all experts.

We use the NCWAA operators to gather decision information from all experts. The group decision matrix $\mathrm{F}$ we obtained is shown as Table 4 .

Step3. Obtain the comprehensive evaluation value

We use the NCWPMSM operator to integrate the evaluation information of different attribute values of each alternative to obtain the comprehensive evaluation value. Here we let $\mathrm{k}_{1}=\mathrm{k}_{2}=2$. The group decision matrix is shown in table 4 .

Step4. Calculate the score function for the alternatives

The score we acquire is $\mathrm{S}_{1}=-0.26, \mathrm{~S}_{2}=-0.24$, $\mathrm{S}_{3}=-0.18, \mathrm{~S}_{4}=-0.14$.

Step5. Rank all the alternatives

Due to $S_{4}>S_{3}>S_{2}>S_{1}$, the ranking of the alternatives is $A_{4}>A_{3}>A_{2}>A_{1}$.

\section{decision-making problem}

To perceive the effect of the parameters $\mathrm{k}_{1}, \mathrm{k}_{2}$ on the decision making, we set different values for the parameters $\mathrm{k}_{1}, \mathrm{k}_{2}$ in step 3 and then rank the alternatives $A_{i}$. The ranking order for different parameters values is given in Table 5 .

Table 5

Ordering of the alternatives based on NCWPMSM by using different values of $\mathrm{k}_{1}, \mathrm{k}_{2}$

\begin{tabular}{ccc}
\hline $\mathrm{k}_{1}, \mathrm{k}_{2}$ & Score values & Ranking order \\
\hline $\mathrm{k}_{1}=1$ & $\mathrm{~S}_{1}=0.1474, \mathrm{~S}_{2}=0.2026$, & $\mathrm{A}_{4}>\mathrm{A}_{3}>\mathrm{A}_{2}>\mathrm{A}_{1}$ \\
$\mathrm{k}_{2}=1$ & $\mathrm{~S}_{3}=0.2516, \mathrm{~S}_{4}=0.2806$ & \\
$\mathrm{k}_{1}=2$ & $\mathrm{~S}_{1}=0.2400, \mathrm{~S}_{2}=0.2517$, & $\mathrm{A}_{4}>\mathrm{A}_{3}>\mathrm{A}_{2}>\mathrm{A}_{1}$ \\
$\mathrm{k}_{2}=1$ & $\mathrm{~S}_{3}=0.2856, \mathrm{~S}_{4}=0.3025$ & \\
$\mathrm{k}_{1}=3$ & $\mathrm{~S}_{1}=-0.8272, \mathrm{~S}_{2}=-0.8423$, & $\mathrm{A}_{4}>\mathrm{A}_{3}>\mathrm{A}_{1}>\mathrm{A}_{2}$ \\
$\mathrm{k}_{2}=1$ & $\mathrm{~S}_{3}=-0.7597, \mathrm{~S}_{4}=-0.7222$ & \\
$\mathrm{k}_{1}=1$ & $\mathrm{~S}_{1}=0.1470, \mathrm{~S}_{2}=0.2019$, & $\mathrm{A}_{4}>\mathrm{A}_{3}>\mathrm{A}_{2}>\mathrm{A}_{1}$ \\
$\mathrm{k}_{2}=2$ & $\mathrm{~S}_{3}=0.2514, \mathrm{~S}_{4}=0.2789$ & \\
$\mathrm{k}_{1}=2$ & $\mathrm{~S}_{1}=-0.2574, \mathrm{~S}_{2}=-0.2383$ & $\mathrm{~A}_{4}>\mathrm{A}_{3}>\mathrm{A}_{2}>\mathrm{A}_{1}$ \\
$\mathrm{k}_{2}=2$ & $\mathrm{~S}_{3}=-0.1800, \mathrm{~S}_{4}=-0.1441$ & \\
$\mathrm{k}_{1}=3$ & $\mathrm{~S}_{1}=-0.8280, \mathrm{~S}_{2}=-0.8438$, & $\mathrm{A}_{4}>\mathrm{A}_{3}>\mathrm{A}_{1}>\mathrm{A}_{2}$ \\
$\mathrm{k}_{2}=2$ & $\mathrm{~S}_{3}=-0.7603, \mathrm{~S}_{4}=-0.7243$ & \\
\hline
\end{tabular}

From Table 5, we can see that when the parameters $\mathrm{k}_{1}$ and $\mathrm{k}_{2}$ take different values, the alternative rankings are roughly the same, and the best alternative is always $\mathrm{A}_{4}$. Therefore, the 
parameters $\mathrm{k}_{1}$ and $\mathrm{k}_{2}$ in the NCWPMSM operator have little influence on decision-making.

\subsection{Comparison with existing neutrosophic cubic fuzzy aggregation operators}

In order to test the effectiveness and superiority of the proposed method, we compared the NCWPMSM operator proposed in this paper with the NCWAA and NCWGA operators provided in reference [29], respectively. The results listed in Table 6 show that the ranking orders based on the NCWPMSM operator is almost the same with the
NCWAA and NCWGA operator and the best alternative is always $A_{4}$, so the NCWPMSM operator is feasible and valid.

Furthermore, only the WPMSM operator considers the situation where the attributes can be divided into different partitions and there is an interrelationship among any attribute in each class, whereas there is no interrelationship among attributes of any two classes. So the NCWPMSM operator has the advantage over the NCWAA and NCWGA operators on aggregating decision information, and it is more effective to handle MAGDM problems under a neutrosophic cubic environment in some cases.

Table 6

Decision results based on different aggregation operators

\begin{tabular}{|c|c|c|c|}
\hline $\begin{array}{l}\text { Aggregation } \\
\text { Operator }\end{array}$ & Aggregated Result & Ranking Order & $\begin{array}{l}\text { The Best } \\
\text { Alternative }\end{array}$ \\
\hline $\begin{array}{l}\text { NCWPMSM } \\
\left(\mathrm{k}_{1}=\mathrm{k}_{2}=2\right)\end{array}$ & $\begin{array}{l}\mathrm{A}_{1}=\{<[0.08,0.13],[0.32,0.36], \\
[0.35,0.38]>,<0.11,0.35,0.36>\} \\
\mathrm{A}_{2}=\{<[0.10,0.13],[0.31,0.35], \\
[0.34,0.37]>,<0.12,0.33,0.36>\} \\
\mathrm{A}_{3}=\{<[0.13,0.18],[0.30,0.35], \\
[0.32,0.36]>,<0.17,0.32,0.34>\} \\
\mathrm{A}_{4}=\{<[0.16,0.23],[0.32,0.35], \\
[0.32,0.36]>,<0.19,0.33,0.33>\}\end{array}$ & $\mathrm{A}_{4}>\mathrm{A}_{3}>\mathrm{A}_{2}>\mathrm{A}_{1}$ & $\mathrm{~A}_{4}$ \\
\hline NCWAA & $\begin{array}{l}\mathrm{A}_{1}=\{<[0.34,0.51],[0.20,0.32], \\
[0.31,0.43]>,<0.31,0.29,0.37>\} \\
\mathrm{A}_{2}=\{<[0.40,0.51],[0.17,0.29], \\
[0.24,0.36]>,<0.22,0.22,0.32>\} \\
\mathrm{A}_{3}=\{<[0.50,0.63],[0.15,0.28], \\
[0.19,0.32]>,<0.21,0.18,0.23>\} \\
\mathrm{A}_{4}=\{<[0.59,0.73],[0.19,0.29], \\
[0.20,0.32]>,<0.25,0.20,0.23>\}\end{array}$ & $\mathrm{A}_{4}>\mathrm{A}_{3}>\mathrm{A}_{2}>\mathrm{A}_{1}$ & $\mathrm{~A}_{4}$ \\
\hline NCWGA & $\begin{array}{l}\mathrm{A}_{1}=\{<[0.33,0.47],[0.21,0.33], \\
[0.32,0.44]>,<0.42,0.31,0.39>\} \\
\mathrm{A}_{2}=\{<[0.40,0.51],[0.17,0.30], \\
[0.25,0.38]>,<0.47,0.22,0.35>\} \\
\mathrm{A}_{3}=\{<[0.48,0.61],[0.17,0.30], \\
[0.24,0.37]>,<0.58,0.21,0.28>\} \\
\mathrm{A}_{4}=\{<[0.56,0.71],[0.22,0.33], \\
[0.24,0.37]>,<0.62,0.25,0.26>\}\end{array}$ & $\mathrm{A}_{4}>\mathrm{A}_{3}>\mathrm{A}_{2}>\mathrm{A}_{1}$ & $\mathrm{~A}_{4}$ \\
\hline
\end{tabular}




\subsection{Comparison with existing score function}

In order to check the validity and superiority of the novel score function, we make a comparison of the proposed score function with the existing score function (Ye 2018). The results of comparison shown in table 7. Obviously, no matter which score function is used, the best alternative is always $A_{4}$, which reflects the effectiveness of the new score function. But in some situations, the existing score function can't distinguish the score valves of alternatives. Such as that when $\mathrm{k}_{1}=\mathrm{k}_{2}=1$, the score values of $A_{4}$ and $A_{3}$ are equal, therefore it cannot evaluate the priority of the alternatives of $\mathrm{A}_{4}$ and $\mathrm{A}_{3}$. But the proposed score function can make up for these shortcomings.

Table 7

Decision results based on different aggregation operators

\begin{tabular}{|c|c|c|}
\hline $2 s$ & & \\
\hline & & \multirow{2}{*}{$A_{4}>A_{3}>A_{2}>A_{1}$} \\
\hline e function & & \\
\hline & & \multirow{2}{*}{$\mathrm{A}_{4}=\mathrm{A}_{3}>\mathrm{A}_{2}>\mathrm{A}_{2}$} \\
\hline & & \\
\hline & & \multirow{2}{*}{$\mathrm{A}_{4}>\mathrm{A}_{3}>\mathrm{A}_{2}>\mathrm{A}_{1}$} \\
\hline & $\mathrm{S}_{3}=$ & \\
\hline & & \multirow{2}{*}{$\mathrm{A}_{4}>\mathrm{A}_{3}>\mathrm{A}_{2}>\mathrm{A}_{2}$} \\
\hline & & \\
\hline & & \multirow{2}{*}{$\mathrm{A}_{4}>\mathrm{A}_{3}>\mathrm{A}_{1}>\mathrm{A}_{2}$} \\
\hline & .72 & \\
\hline 5 & & \multirow{2}{*}{$\mathrm{A}_{4}=\mathrm{A}_{3}>\mathrm{A}_{1}=\mathrm{A}_{3}$} \\
\hline & & \\
\hline & & \multirow{2}{*}{$\mathrm{A}_{4}>\mathrm{A}_{3}>\mathrm{A}_{2}>\mathrm{A}_{1}$} \\
\hline & $\mathrm{S}_{3}=0$ & \\
\hline exiting & $\begin{array}{l}\mathrm{S}_{1}=0.6 \\
\mathrm{~S}_{3}=0.6\end{array}$ & $\mathrm{~A}_{4}>\mathrm{A}_{3}>\mathrm{A}_{2}>\mathrm{A}_{2}$ \\
\hline nc & $\begin{array}{l}S_{1}=-0 \\
S_{3}=-0\end{array}$ & $\mathrm{~A}_{4}>\mathrm{A}_{3}>\mathrm{A}_{2}>\mathrm{A}_{1}$ \\
\hline $\mathrm{k}_{2}=2$ score & $\begin{array}{l}\mathrm{S}_{1}=0.47, \mathrm{~S}_{2}=0.48 \\
\mathrm{~S}_{3}=0.50, \mathrm{~S}_{4}=0.51\end{array}$ & $\mathrm{~A}_{4}>\mathrm{A}_{3}>\mathrm{A}_{2}>\mathrm{A}_{2}$ \\
\hline $\mathrm{n}$ & $\begin{array}{l}\mathrm{S}_{1}=-0.83, \mathrm{~S}_{2}=-0.84, \\
\mathrm{~S}_{3}=-0.76, \mathrm{~S}_{4}=-0.72\end{array}$ & $\mathrm{~A}_{4}>\mathrm{A}_{3}>\mathrm{A}_{1}>\mathrm{A}_{2}$ \\
\hline $2=2 \quad$ exiting & $\begin{array}{c}\mathrm{S}_{1}=0.10, \mathrm{~S}_{2}=0.10 \\
\mathrm{~S}_{3}=0.14, \mathrm{~S}_{4}=0.14\end{array}$ & $\mathrm{~A}_{4}=\mathrm{A}_{3}>\mathrm{A}_{1}=\mathrm{A}$ \\
\hline
\end{tabular}

\section{Conclusion}

In this paper, firstly, we put the partitioned Maclaurin symmetric mean operator to neutrosophic cubic environment and propose the neutrosophic cubic partitioned Maclaurin symmetric mean (NCPMSM) operator and neutrosophic cubic weighted partitioned Maclaurin symmetric mean (NCPMSM) operator. Then, we verify some characteristics for the proposed operators. Later, we extend the score function of IFV to neutrosophic cubic environment and define a novel score function, which can overcome the shortcomings of existing score functions. Finally we developed a new approach for MAGDM problems with NCWPMSM operator and the novel score function in neutrosophic cubic environment, and applying an example of supplier selection to this method. By setting different parameters in NCWPMSM operator, we know that the best alternative remains unchanged when the parameters are changed due to subjective preferences. By comparing with existing operators and score function, we verify the effectiveness and superiority of the proposed operator and novel function.

In the future, it is necessary to apply the NCWPMSM operator to other fields, such as medical diagnosis, pattern recognition and decision support. Besides, it is necessary to improve the proposed novel score function.

Acknowledgements This work was supported in part by the Fund for Shanxi "1331 Project" Key Innovative Research Team (TD201710), and in part by "The Discipline Group Construction Plan for Serving Industries Innovation", Shanxi, China: The Discipline Group Program of Intelligent Logistics Management for Serving Industries Innovation (XKQ201801).

\section{Compliance with ethical standards}

Conflict of interest All the authors declare that they do not have any conflict of interest. 
Ethical Approval This article does not contain any studies with human participants or animals performed by any of the authors.

Author Contributions Funding acquisition, Mei Qin $\mathrm{Wu}$; Methodology, Jian Ping Fan; Writingoriginal draft, Shanshan Zhai; Writing-review \& editing, Shanshan Zhai.

\section{References}

Zadeh LA (1965) Fuzzy sets. Information Control 8(3):338-353

Atanassov KT (1986) Intuitionistic fuzzy sets, Fuzzy Sets Systems 20(1):87-96

Atanassov KT (1989) More on intuitionistic fuzzy sets, Fuzzy Sets Systems 33(1):33-37

Atanassov KT, Gargov G (1989) Interval-valued intuitionistic fuzzy sets, Fuzzy Sets Systems 31(3):343-349

Smarandache F (1999) An unifying field in logics. neutrosophy: Neutrosophic probability, set and logic. Rehoboth: American Research Press:1-141

Wang HB, Madiraju P, Zhang YQ, Sunderraman R (2005) Interval Neutrosophic Sets. International Journal of Applied Mathematics and Statistics 3(5):1-18

Wang HB., Smarandache F, Zhang YQ, Sunderraman R (2010) Single Valued Neutrosophic Sets. Review of the Air Force Academy 1:10-14

Jun YB, Kim CS, Yang KO (2011) Cubic sets. Annals of Fuzzy Mathematics and Informatics 4(1):83-98

Turksen IB (1986) Interval valued fuzzy sets based on normal forms. Fuzzy Sets and Systems 20(2): 191-210

Mehmood F, Mahmood T, Khan Q (2016) Cubic hesitant fuzzy sets and their applications to multi criteria decision making. International Journal of Algebra and Statistics 5(1):19-51

Muhiuddin G, Al-Roqi AM (2014) Cubic soft sets with applications in BCK/BCI-algebras. Ann Fuzzy Math Inform 8(2):291-304

Aslam M, Fahmi A (2020) New work of trapezoidal cubic linguistic uncertain fuzzy Einstein hybrid weighted averaging operator and decision making. Soft Computing 24 3331-3354

Amin F, Fahmi A, Aslam M (2020) Approaches to multiple attribute group decision making based on triangular cubic linguistic uncertain fuzzy aggregation operators. Soft Computing 24:11511-11533

Jun YB, Smarandache F, Kim CS, Neutrosophic cubic sets. New Mathematics and Natural Computation 13(1):41-54

Gulistan M., Beg I, Yaqoob N (2018) Decision making problems under the environment of neutrosophic cubic soft matrices. Journal of Intelligent and Fuzzy Systems:1-13

Molodtsov D (1999) Soft set theory - First results. Computers and Mathematics with Applications 37(4):19-31

Gulistan M, Khan S (2019) Extentions of neutrosophic cubic sets via complex fuzzy sets with application. Complex and Intelligent Systems 6(2):309-320
Cui WH, Ye J (2019) Logarithmic similarity measure of dynamic neutrosophic cubic sets and its application in medical diagnosis. Computers in Industry 111:198-206

Maclaurin C (1729) A second letter to Martin Folkes, Esq; concerning the roots of equations, with demonstration of other rules of algebra. Philos Trans R Soc London Ser A 36:59-96

Qin JD, Liu XW (2014) An approach to intuitionistic fuzzy multiple attribute decision making based on Maclaurin symmetric mean operators, Journal of Intelligent and Fuzzy Systems 27(5):2177-2190

Li W, Zhou XQ, Guo GQ (2016) Hesitant fuzzy Maclaurin symmetric mean operators and their application in multiple attribute decision making, Computational analysis and applications 20(1):459-469

Wang JQ, Yang Y, Li L (2018) Multi-criteria decision-making method based on single-valued neutrosophic linguistic Maclaurin symmetric mean operators. Neural Computing and Applications 30(5):1529-1547

Qin JD, Liu XW (2015) Approaches to uncertain linguistic multiple attribute decision making based on dual Maclaurin symmetric mean. Journal of Intelligent and Fuzzy Systems 29(1):171-186

Bai KY, Zhu XM, Wang J, Zhang RT (2018) Some partitioned maclaurin symmetric mean based on q-rung orthopair fuzzy information for dealing with multi-attribute group decision making. Symmetry 10(9):383

Zeng SZ, Chen MS, Kuo LW (2019) Multiattribute decision making based on novel score function of intuitionistic fuzzy values and modified VIKOR method. Information Sciences 488:76-92

Pramanik S, Dey PP, Giri BC, Smarandache F (2017) An extended TOPSIS for multi-attribute decision making problems with neutrosophic cubic information. Neutrosophic Sets and Systems 17:20-28

Ali M, Deli I, Smarandache F (2016) The theory of neutrosophic cubic sets and their applications in pattern recognition. Journal of Intelligent and Fuzzy Systems 30(4):1957-1963

Liu PD, Khan Q, Mahmood T (2020) Group decision making based on power Heronian aggregation operators under neutrosophic cubic environment. Soft Computing 24:19711997

Ye J (2018) Operations and aggregation method of neutrosophic cubic numbers for multiple attribute decision-making, Soft Computing 22(22):7435-7444

Nguyen H (2020) Some new operations on Atanassov's intuitionistic fuzzy sets in decision-making problems. Journal of Intelligent and Fuzzy Systems 38:639-651

Liu PD, Chen SM, Wang YM (2020) Multiattribute group decision making based on intuitionistic fuzzy partitioned Maclaurin symmetric mean operators. Information Sciences 512:830-854

Liu PD, You XL (2020) Linguistic neutrosophic partitioned Maclaurin symmetric mean operators based on clustering algorithm and their application to multi-criteria group decision-making. Artificial Intelligence Review 53(3):2131-2170

Liu PD, Li Y (2020) Multiattribute decision method for comprehensive logistics distribution center location 
selection based on 2-dimensional linguistic information. Information Sciences 538:209-244. 